\title{
Free Fermions and the Alexander-Conway Polynomial
}

\author{
L. H. Kauffman ${ }^{1, \star}$ and H. Saleur ${ }^{2, \star \star}$ \\ 1 Department of Mathematics, Statistics and Computer Science, \\ The University of Illinois at Chicago, Chicago, IL 60680, USA

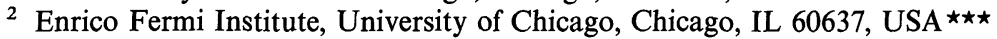 \\ Received September 20, 1990; in revised form December 28, 1990
}

\begin{abstract}
We show how the Conway Alexander polynomial arises from the $q$ deformation of $\left(Z_{2}\right.$ graded) $s l(n, n)$ algebras. In the simplest $s l(1,1)$ case we then establish connection between classical knot theory and its modern versions based on quantum groups. We first show how the crystal and the fundamental group of the complement of a knot give rise naturally to the Burau representation of the braid group. The Burau matrix is then transformed into the $U_{q} s l(1,1) R$ matrix by going to the exterior power algebra. Using a det $=$ str identity, this allows us to recover the state model of $[\mathrm{K} 2,89]$ as well. We also show how the $U_{q} s l(1,1)$ algebra describes free fermions "propagating" on the knot diagram. We rewrite the Conway Alexander polynomial as a Berezin integral, and thus as an apparently new determinant.
\end{abstract}

\section{Introduction}

This paper discusses the role of the Alexander-Conway polynomial [A23, C70] in relation to quantum groups and statistical mechanics. In particular, we show that a state model for this polynomial is identical in form to the free-fermion model in statistical mechanics. This means that the polynomial can be expressed as a discrete Berezin integral, and through this as a determinant that is distinct from the classical determinant definition of this polynomial.

A number of significant interconnections arise from our work. The YangBaxter solution in the $s l(1 / 1)$ case is seen to arise directly from the Burau representation of the Artin braid group (via its action on exterior powers). Thus we provide a direct line from the classical knot theory to the statistical mechanics. The

\footnotetext{
$\star$ Work supported in part by NSF grant no. DMS- 8822602

$\star \star$ Work supported in part by the NSF: grant nos. PYI PHY 86-57788 and PHY 90-00386 and by CNRS, France

$\star \star \star$ On leave from SPhT CEN Saclay, F-91191 Gif sur Yvette, Cedex, France
} 
algebraic key to this connection is a formula (proved in the Appendix) that connects determinant and trace by expressing the determinant $\operatorname{det}(A+s I)$ as a trace: $\operatorname{det}(A+s I)=\operatorname{tr}(\mathscr{S} \hat{A})$, where $\hat{A}$ is the extension of $A: V \rightarrow V$ to $\hat{A}: A^{*}(V)$ $\rightarrow \Lambda^{*}(V)$, where $\Lambda^{*}(V)$ denotes the exterior algebra of $V$ and $\mathscr{S}(\hat{v})=s^{k} \hat{v}$ when $\hat{v} \in \Lambda^{*}(V)$ has degree $k$. A chart of these relationships is shown in Fig. 0 .

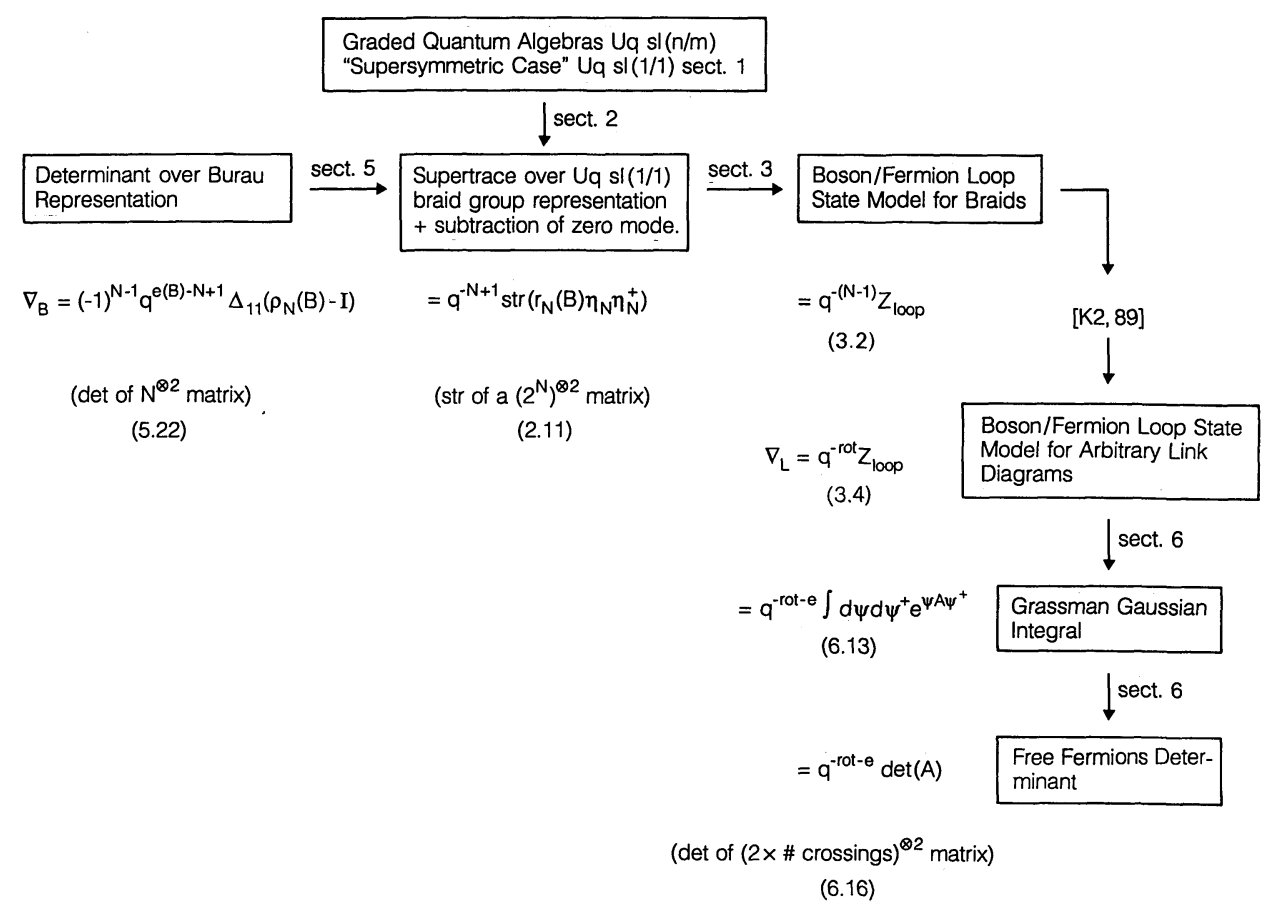

Fig. 0. From Burau to free fermions flow chart

The history of statistical mechanics models for the Alexander-Conway polynomial is an interesting matter. In [K83] (see also [K1, 89]) the first author showed how to modify Alexander's original definition to obtain a model for the Conway-Alexander polynomial as a state summation. This model can be regarded as the zero-temperature limit of a generalized Potts model, but it does not seem to have a naturally associated solution to the Yang-Baxter equation. In [K87] and [K88] the first statistical mechanics models for the Jones polynomial appeared. It was then seen that these models for the original Jones polynomial were part of a much wider class of models for knot polynomials based on solutions to the Yang-Baxter equation [AW87, T88, Jo89]. However, it took a little time before it became apparent [LCS88, Ja87, K2, 89] that there was a Yang-Baxter model for the Alexander-Conway polynomial itself. These models [Ja87, K2, 89] are technically distinct from the other models in two ways: As discussed in $[\mathrm{K} 2,89]$ and in this paper, the associated quantum group is not strictly classical and the model itself is a correlation function rather than a straight summation over states of the link diagram. This last technicality is related to the fact that for the ConwayAlexander polynomial $\nabla_{K}$, we have $\nabla_{K \amalg K^{\prime}}=0$ when $K \amalg K^{\prime}$ is a disjoint union of links $K$ and $K^{\prime}$. This means that $\nabla_{0 \amalg K}=0$, thus the "loop value" in this model is 
zero. In order to avoid the contradiction $\nabla_{0}=0$, we represent links by two-strand tangles, so that the unknot is represented by an unknotted strand $\longrightarrow$ with $\nabla \nrightarrow=1$. In this picture a trefoil has the form

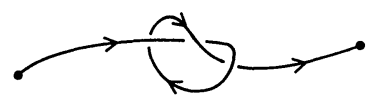

and ambient isotopy is taken relative to the endpoints of the tangle. With these conventions, it is easy to set up the statistical mechanics models so that

$$
\nabla_{0}=0, \quad \nabla \leadsto=1 \text {. }
$$

It should also be remarked that the Alexander-Conway polynomial (in the form above) is characterized by the $\nabla_{K_{+}}-\nabla_{K_{-}}=z \nabla_{K_{0}}$ skein relation, where $K_{+}, K_{-}$, and $K_{0}$ are a triple of links that differ at a single crossing as shown below:

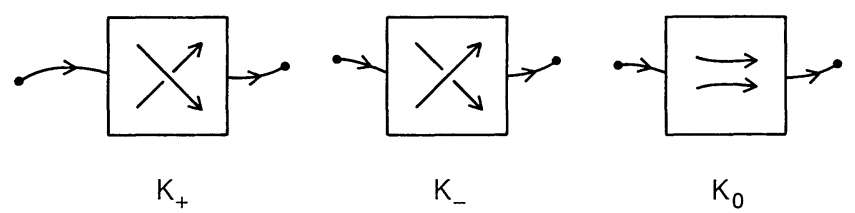

That is, a positive crossing in $K_{+}$is switched to form a negative crossing in $K_{-}$, and the crossing is spliced out to form $K_{0}$. It was Conway's insight that this polynomial can be defined entirely by diagrammatic properties, an insight that paved the way for the blossoming of knot theory through the Jones polynomial, its generalizations, and interconnections with mathematical physics.

Finally, we must point out that our formula for the Alexander-Conway polynomial as a fermionic integral:

$$
\nabla_{\boldsymbol{K}}=q^{-r o t-e} \int d \psi d \psi^{+} \exp \left(\psi A \psi^{+}\right)
$$

(see Sect. 6.13) raises the question of the relationship of these discrete methods with the approach of Witten [W89] via quantum field theory. A supersymmetric extension of Witten's theory may capture the Alexander-Conway polynomial.

The paper is organized as follows: We discuss in Sect. 1 the $q$ deformation of $s l(n / m)$ graded algebras and the associated $R$ matrices in the fundamental representation. We show that the resulting link invariants obtained via representation of the braid group satisfy the skein relation (1.39) with $k=n-m$. The Conway-Alexander polynomial is thus (formally) recovered in the balanced ("supersymmetric") case $n=m$.

The algebras $U_{q} s l(n / m)$ or $U_{q} g l(n / m)$ have also been studied in [CO89, CK90]. Link invariants derived from graded algebras appear also in [LCS88]. The connection between the Conway polynomial and $U_{q} s l(1 / 1)$ (or "free fermions") seems to have been noticed independently by several authors [D89, R89, S89].

Section 2 deals with the regularization of Sect. 1 expressions in the balanced case $\operatorname{sl}(1 / 1)$. We show how to extract the Conway-Alexander polynomial from the $U_{q} s l(1 / 1) R$ matrix by subtracting a zero mode or considering tangles.

In Sect. 3 we show how the supertrace over the $U_{q} s l(1 / 1)$ braid group representation expands into a state model involving bosonic and fermionic loops. 
Connection is made with the state model of $[\mathrm{K} 2,89]$, which allows extension of the formalism to arbitrary link diagrams.

Section 4 is an intermezzo in classical knot theory.

Section 5 elucidates the relation between the Burau representation and the $U_{q} s l(1 / 1)$ model. Since the Burau representation describes one particle fermionic states carried on strands, we introduce a multiparticle model by going to the exterior algebra. This model coincides with the $U_{q} s l(1 / 1)$ model through a simple $\mathrm{det}=\mathrm{str}$ identity. Besides the Yang-Baxter property which means factorization of multiparticle scattering on two particle terms, the $U_{q} s l(1 / 1) R$ matrix enjoys factorization of two particle scattering onto one particle terms. This justifies the "free fermion" denomination.

In Sect. 6 we show that the state model partition function can be rewritten as a Gaussian integral. The absence of four fermions interaction term is another manifestation of the free fermion property. The tangle diagram can thus be considered as a "Feynman graph" in a fermionic theory, and the AlexanderConway polynomial as a correlation function. Finally the integral is recast in an apparently new determinant expression for the Conway polynomial.

\section{Quantum $\operatorname{sl}(n / m)$}

The superalgebra $\operatorname{sl}(n / m)[\mathrm{Ka} 77, \mathrm{Sc} 79]$ is the algebra of $(n+m) \times(n+m)$ matrices with vanishing supertrace in a supervector space of superdimension $n-m$. In an homogeneous basis $(p(i)=0, i=1 \ldots n ; p(i)=1, i=n+1 \ldots n+m)$ elements of $s l(n / m)$ read

$$
X=\left(\begin{array}{c|c}
\stackrel{m}{A} & \stackrel{m}{B} \\
\hline C & D
\end{array}\right) \begin{aligned}
& n \\
& m
\end{aligned}, \quad \operatorname{str} X=\operatorname{tr} A-\operatorname{tr} D=0 .
$$

Hence $s l(n / m)$ has even part $s l(n) \otimes s l(m) \otimes U(1)$ with $n^{2}+m^{2}-1$ even generators, and $2 m n$ odd generators. Its superdimension is

$$
s \operatorname{dim} s l(n / m)=(n-m)^{2}-1 .
$$

The Cartan subalgebra has dimension $n+m-1$ with the $n-1$ first generators $h_{i}$ belonging to $\operatorname{sl}(n)$, the $m-1$ last to $\operatorname{sl}(m)$, while $h_{n}$ plays a special role. All $h$ 's are even. Setting

$$
\left(E_{i j}\right)_{k l}=\delta_{i k} \delta_{j l}
$$

one has

$$
\begin{gathered}
h_{i}=E_{i i}-E_{i+1, i+1}, \quad i=1 \ldots n-1, \quad n+1 \ldots n+m-1, \\
h_{n}=E_{n n}+E_{n+1, n+1} .
\end{gathered}
$$

To each $h_{i}(i \neq n)$ corresponds a raising operator $e_{i}$ and a lowering one $f_{i}$. Both are even

$$
e_{i}=E_{i, i+1}, \quad f_{i}=E_{i+1, i} .
$$

To $h_{m}$ corresponds a pair of odd operators

$$
\eta=E_{n, n+1}, \quad \eta^{+}=E_{n+1, n} .
$$


The calculation of super Lie bracket using (1.3-1.6) gives

$$
\begin{cases}{\left[e_{i}, f_{j}\right]=\delta_{i j} h_{i}} & i, j=1 \ldots n-1, n+1 \ldots n+m-1, \\ {\left[\eta, \eta^{+}\right]_{+}=h_{m}} & \eta^{2}=\eta^{+2}=0, \\ {\left[\eta, f_{i}\right]=\left[e_{i}, \eta^{+}\right]=0} & i \neq n, \\ {\left[h_{i}, e_{j}\right]=a_{i j} e_{j}} & \text { for any } i, j\left(e_{n} \equiv \eta\right), \\ {\left[h_{i}, f_{j}\right]=-a_{i j} f_{j}} & \text { for any } i, j\left(f_{n} \equiv \eta^{+}\right),\end{cases}
$$

where $a_{i j}$ are elements of the Cartan matrix of $s l(n / m)$

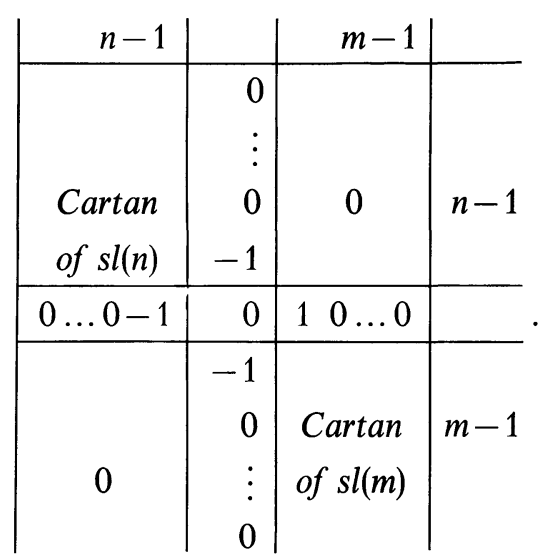

Equation (1.7) defines then the abstract algebra $s l(n / m)$ in terms of simple roots. The preceding $(n+m) \times(n+m)$ matrix representation is then the fundamental representation of this algebra. Finally we notice the $U(1)$ generator

$$
Q=\sum_{i=1}^{n-1} \frac{i h_{i}}{n}+h_{n}-\sum_{i=1}^{m-1} \frac{m-i}{m} h_{n+i}
$$

which reads in the fundamental representation

$$
Q=\left(\begin{array}{c|c}
\frac{1}{n} \mathbf{1}_{n} & 0 \\
\hline 0 & \frac{1}{m} 1_{m}
\end{array}\right) .
$$

If $n=m, Q=\frac{1}{n} 1$. In this case $s l(n / n)$ is not simple. To get a simple algebra one has to take the coset by the ideal $\lambda 1$. In Kac notations [Ka77],

$$
\begin{gathered}
A(n, m)=s l(n+1, m+1) \quad n \neq m, \\
A(n, n)=s l(n+1, n+1) / \lambda \mathbf{1}_{2 n+2}
\end{gathered}
$$

with $s \operatorname{dim} A(n, n)=-2$.

We then define the quantum deformation $U_{q} s l(n / m)$ as the associative superalgebra (with unit) generated by $e_{i}, f_{i}, h_{i}, \eta, \eta^{+}, h_{n}$ with relations [CK90, 
KR89, S90]

$$
\begin{cases}{\left[e_{i}, f_{j}\right]=\delta_{i j}\left(h_{i}\right)_{q}} & i, j=1 \ldots n-1, n+1 \ldots n+m-1 \\ {\left[\eta, \eta^{+}\right]_{+}=\left(h_{n}\right)_{q}} & \eta^{2}=\eta^{+2}=0 \\ {\left[\eta, f_{i}\right]=\left[e_{i}, \eta^{+}\right]=0,} & \\ {\left[h_{i}, e_{j}\right]=a_{i j} e_{j}} \\ {\left[h_{i}, f_{j}\right]=-a_{i j} f_{j},}\end{cases}
$$

where

$$
(n)_{q}=\frac{q^{n}-q^{-n}}{q-q^{-1}}
$$

Only $\left[e_{i}, f_{j}\right]$ and $\left[\eta, \eta^{+}\right]_{+}$are modified with respect to (1.7). $U_{q} s l(n / m)$ is a deformation of the universal enveloping algebra $U s l(n / m)$. The fundamental representations $\square$ of $U_{q} s l(n / m)$ and $U s l(n / m)$ coincide since $( \pm 1)_{q}= \pm 1$. The (graded) tensor product can be defined using the superalgebras homomorphism $\Delta: U_{q} \rightarrow U_{q} \otimes U_{q}$ such that

$$
\begin{cases}\Delta\left(h_{i}\right)=h_{i} \otimes 1+1 \otimes h_{i}, & i=1 \ldots n+m-1, \\ \Delta\left(e_{i}\right)=q^{h_{i} / 2} \otimes e_{i}+e_{i} \otimes q^{-h_{i} / 2}, & i=1 \ldots n-1, \\ \Delta\left(f_{i}\right)=q^{h_{i} / 2} \otimes f_{i}+f_{i} \otimes q^{-h_{i} / 2}, & i=1 \ldots n-1, \\ \Delta(\eta)=q^{h_{n} / 2} \otimes \eta+\eta \otimes q^{-h_{n} / 2}, & \\ \Delta\left(\eta^{+}\right)=q^{h_{n} / 2} \otimes \eta^{+}+\eta^{+} \otimes q^{-h_{n} / 2}, \\ \Delta\left(e_{i}\right)=q^{-h_{i} / 2} \otimes e_{i}+e_{i} \otimes q^{h_{i} / 2}, & i=n+1 \ldots n+m-1, \\ \Delta\left(f_{i}\right)=q^{-h_{i} / 2} \otimes f_{i}+f_{i} \otimes q^{h_{i} / 2}, & i=n+1 \ldots n+m-1 .\end{cases}
$$

[Recall that in a graded tensor product $(a \otimes b) \cdot(v \otimes w)=(-)^{p(b) p(v)} a \cdot v \otimes b \cdot w$, where $a, b$ are operators and $v, w$ vectors.] A remarkable feature of formulas (1.14) is that the coproduct of the first $n-1$ raising or lowering operators is built with $q$ while the coproduct of the last $m-1$ ones uses $q^{-1}$ instead. This contrasts with the $s l(n+m)$ situation where all coproducts would involve $q$ only, and originates in that $h_{n}=E_{n n}-E_{n+1, n+1}$ in the $\operatorname{sl}(n+m)$ case while $h_{n}=E_{n n}+E_{n+1, n+1}$ in the $\operatorname{sl}(n / m)$ case. Of course besides $\Delta$ the other coproduct $\bar{\Delta}$ is possible where $q$ is replaced by $q^{-1}$ in (1.14). $\Delta$ and $\bar{\Delta}$ are related by a universal $\mathscr{R}$ matrix [D86]

$$
\mathscr{R} \Delta=\bar{\Delta} \mathscr{R}
$$

which satisfies the graded Yang-Baxter equation. Introducing the graded permutation operator

$$
\mathscr{P} v \otimes w=(-)^{p(v) p(w)} w \otimes v
$$

the matrix $\check{R}=\mathscr{P} \mathscr{R}$ satisfies

$$
\check{R}_{12} \check{R}_{23} \check{R}_{12}=\check{R}_{23} \check{R}_{12} \check{R}_{23}
$$

which written in components looks the same as in the non-graded case [KS82, S90]. Although we do not know the general expression for $\mathscr{R}$, it is easy to find the 
expression of $\check{R}$ acting in the tensor product of two fundamental representations,

$$
\begin{aligned}
\check{R}= & \sum_{i=1}^{n} q E_{i i} \otimes E_{i i}-\sum_{i=n+1}^{n+m} q^{-1} E_{i i} \otimes E_{i i} \\
& +\sum_{i<j}\left(q-q^{-1}\right) E_{i i} \otimes E_{j j}+\sum_{i \neq j}(-)^{p(i) p(j)} E_{i j} \otimes E_{i j} .
\end{aligned}
$$

This coincides with the graded $R$ matrices of [SC81, DA90]. For $s l(1 / 1)$ one gets (this matrix appeared in connection with $s l(1 / 1)$ in [S89, SVZ89])

$$
\breve{R}=\left(\begin{array}{cccc}
q & 0 & 0 & 0 \\
0 & q-q^{-1} & 1 & 0 \\
0 & 1 & 0 & 0 \\
0 & 0 & 0 & -q^{-1}
\end{array}\right)
$$

graphically

$$
\left.\left.\check{R}=\left(q-q^{-1}\right)\right\rangle_{i}<\bigwedge_{j}+(-)^{p(i)} q^{(-)^{p(i)}}\right\rangle_{i}=\prod_{i}+(-)^{p(i) p(j)} \searrow_{i}^{\neq}
$$

The $(-)^{p(i) p(j)}$ terms in (1.18) can be replaced by 1 without affecting the YangBaxter equation (gauge transformation). $\check{R}$ looks then like the $U_{q} s l(n+m)$ solution except that diagonal terms have the value $-q^{-1}$ instead of $q$. These two coincide for $q=i$ which is a higher symmetry point. We can draw schematically

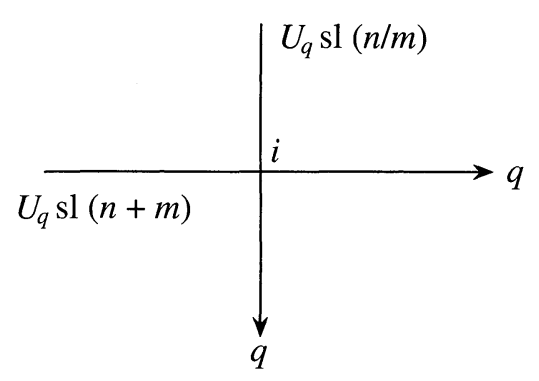

If $q \rightarrow 1, \breve{R} \rightarrow \mathscr{P}$. Besides (1.17), $\breve{R}$ satisfies a quadratic relation

$$
\check{R}=\check{R}^{-1}+q-q^{-1} \text {. }
$$

Hence working in the tensor product of $N$ fundamental representations and setting $r_{a}=\breve{R}_{a, a+1} a=1 \ldots N-1$ we get with $(1.17,1.21)$ a representation of the Hecke algebra $H_{N}[\mathrm{~J} 86]$

$$
\left\{\begin{array}{l}
r^{2}=1+\left(q-q^{-1}\right) r \\
r_{a} r_{a \pm 1} r_{a}=r_{a \pm 1} r_{a} r_{a \pm 1} \\
{\left[r_{a}, r_{b}\right]=0,|a-b| \geqq 2}
\end{array}\right.
$$

Due to (1.15), $\check{R}$ commutes with the quantum superalgebra

$$
\left[\check{R}, U_{q} s l(n / m)\right]=0 \text {. }
$$


Accordingly $\check{R}$ reads for $q$ generic

$$
\check{R}=q P_{\square}-q^{-1} P_{\text {日 }}
$$

with

$$
\begin{array}{r}
\operatorname{dim} \square=\frac{(n+m)^{2}+n-m}{2} \\
\operatorname{dim} \square=\frac{(n+m)^{2}+m-n}{2}
\end{array}
$$

in the balanced case $n=m$, the eigenvalues $q$ and $-q^{-1}$ occur with the same degeneracy $2 n^{2}$. As for non-graded algebras, the case $q$ a root of \pm 1 requires some care. If $q=i$ for instance, $R$ is not diagonalizable. All zeros of the characteristic polynomial are equal to $i$ but obviously $\breve{R} \neq i$. The two representations $\square$ and $\boxminus \mathrm{mix}$ in a larger representation [PS90] that is indecomposable but not irreducible. In what follows we suppose $q$ generic.

For $n \neq m$, introducing the quadratic Casimir eigenvalues [BB80],

$$
\begin{aligned}
c_{\square} & =\frac{(n-m)^{2}-1}{2(n-m)}, \\
c_{\square} & =\frac{(n-m)^{2}+(n-m)-2}{n-m}, \\
c_{\boxminus} & =\frac{(n-m)^{2}-(n-m)-2}{n-m},
\end{aligned}
$$

we can rewrite $(1.18)$ as

$$
\check{R}=q^{\frac{1}{n-m}}\left(q^{c \varpi-2 c \square} P_{\square}-q^{c 日^{-2 c} \square} P_{日}\right) .
$$

This formal expansion coincides with the $s l(n-m)$ one.

In the graded case, since antisymmetrization over even coordinates implies symmetrization over odd ones, there can be Young diagrams with an arbitrary number of rows. We therefore do not expect $\check{R}$ to satisfy besides $(1.17,1.21)$ the kind of additional relations encountered in the non-graded case.

Because $\check{R}$ obeys the Hecke algebra, a solution of the spectral parameter dependent Yang-Baxter equation is obtained by [J85, J86]

$$
\check{R}(u)=\left(q y^{-1}-q^{-1} y\right) \mathbf{1}+\left(y-y^{-1}\right)\left(q^{-1}-\check{R}\right),
$$

where $y=e^{i u}$ and

$$
\check{R}_{12}(u) \check{R}_{23}(u+v) \check{R}_{12}(v)=\check{R}_{23}(v) \check{R}_{12}(u+v) \check{R}_{23}(u) .
$$

We introduce a set of vectors $\Lambda_{i}, i=1 \ldots n+m-1$ and the Weyl vector $\varrho$ with

$$
\begin{gathered}
\Lambda_{i} \cdot \varrho=\frac{(n-m) i-i^{2}}{2} i=1, \ldots, n, \\
\Lambda_{i} \cdot \varrho=\frac{(n-m)(i-n-m)-(i-n-m)^{2}}{2} i=n+1 \ldots n+m-1 .
\end{gathered}
$$


The super $q$-dimension of a representation $r$ is then defined as

$$
\begin{aligned}
s q \operatorname{dim} r & =\operatorname{str}_{r} q^{2 \varrho \cdot\left[\sum_{i=1}^{n} h_{i} \Lambda_{i}-\sum_{i=n+1}^{n+m-1} h_{i} \Lambda_{i}\right]} \\
& \stackrel{\text { def }}{\equiv} \operatorname{str}_{r} q^{H},
\end{aligned}
$$

In the fundamental representation

$$
s q \operatorname{dim} \square=(n-m)_{q} .
$$

Formula (1.32) is reminiscent of $\operatorname{sl}(n / m)$ one. In the graded case we take a supertrace instead. Moreover $h_{i}, i=1 \ldots n$ come with a factor $q$ while $h_{i}, i=n+1 \ldots n+m-1$ come with $q^{-1}$, as in coproduct formulas (1.14).

One has

$$
\begin{aligned}
\operatorname{str} q^{H} \check{R} & =q^{n-m}(n-m)_{q}, \\
\operatorname{str} q^{H} \check{R}^{-1} & =q^{-(n-m)}(n-m)_{q} .
\end{aligned}
$$

For $n \neq m$ we introduce the super Markov trace [WDA89]

$$
s \operatorname{Tr} X=\frac{1}{(n-m)_{q}^{N}} \operatorname{str} q^{H} X,
$$

where $X$ is an operator in the $H_{N}$ algebra. It satisfies the Markov properties

$$
\left\{\begin{array}{l}
s \operatorname{Tr} X Y=s \operatorname{Tr} Y X \\
s \operatorname{Tr} X r_{N}=\tau s \operatorname{Tr} X \\
s \operatorname{Tr} X r_{N}^{-1}=\tau^{-1} s \operatorname{Tr} X
\end{array},\right.
$$

where $\tau=q^{n-m} /(n-m)_{q}$. The proof of formulas (1.32-1.36) is a bit lengthy. It will appear in [KS91]. In the following we use results for $n=m=1$ only. In this case the validity of $(1.32-1.36)$ is easily checked using the state model.

The $r_{a}$ operators (1.22) provide a representation of the braid group

$$
B_{N}: r_{a}=r_{N}\left(\sigma_{a}\right) \text {. }
$$

Recall that $B_{N}$ is generated by elementary braids $\sigma_{1}, \sigma_{2}, \ldots, \sigma_{N-1}$ and their inverses, with $\sigma_{a}$ corresponding to a positive crossing of the $a^{\text {th }}$ and $(a+1)^{\text {th }}$ strands of an otherwise undisturbed braid. We shall represent braids with an upward orientation. Thus $\sigma_{1} \sigma_{2} \sigma_{1}$ is shown as

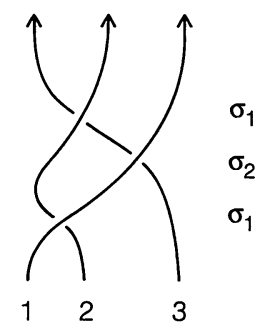

with braid strands labelled 1, 2, 3 for three-strand braids. With the super Markov trace we therefore have at hand all ingredients to build link polynomials. Consider a link $\bar{B}$ closure of some braid $B$ on $N$ strands. (The closure of a braid is obtained by 
attaching the top and bottom strands in parallel fashion as is shown in Fig. 1.) An invariant is obtained as

$$
P_{\bar{B}}=(\tau \bar{\tau})^{-(N-1) / 2}\left(\frac{\bar{\tau}}{\tau}\right)^{e(B) / 2} s \operatorname{Tr} r_{N}(B)
$$

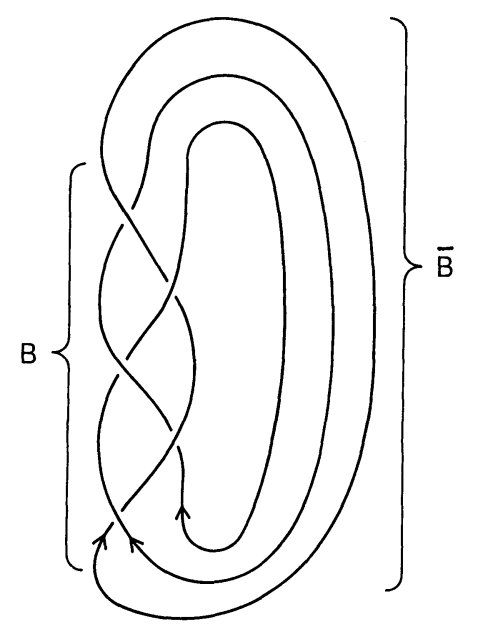

Fig. 1. Closure of the braid $B=\sigma_{1}^{-1} \sigma_{2} \sigma_{1}^{-1} \sigma_{2} \sigma_{1}^{-1}$

where $r_{N}(B)$ is the braid operator in the $U_{q} s l(n / m)$ fundamental representation case and $e(B)$ is the sum of exponents. Using (1.21) one gets the Skein relation.

$$
P_{\lambda}=\left(q-q^{-1}\right)\left(\frac{\bar{\tau}}{\tau}\right)^{1 / 2} P_{\eta+}+\frac{\bar{\tau}}{r} P_{X}
$$

or

$$
q^{n-m} P_{\lambda}-q^{-(n-m)} P_{X}=\left(q-q^{-1}\right) P_{) \uparrow}
$$

Many properties of $U_{q} s l(n / m)$ have the same algebraic form as those of $U_{q} s l(n-m)$. This thus provides a natural framework to give a meaning to "sl(1), $s l(0)$ or $s l(-n)$ ". For instance an explicit braid group like calculation of the Conway-Alexander polynomial should be possible using any of the $U_{q} s l(n / n)$ algebras. Without the introduction of fermions one would need to make the calculation for the general $U_{q} s l(N)$ case, and then continue analytically to $N=0$.

As a first example of application consider the link polynomial built with $U_{q} s l(2 / 1)$. In this case the fundamental representation has a super $q$ dimension equal to $1 . \square^{\otimes N}$ decomposes onto a sum of representations each having zero super $q$ dimensions except for $\square\rceil \ldots \square$ which has super $q$ dimension 1 . On the latter obviously $s \operatorname{Tr} B=q^{e(B)}$. Using (1.37) we get $P=1$ for any link. This agrees with the analysis of [LiM87] based on the Skein relation:

$$
q P_{X}-q^{-1} P_{X}=\left(q-q^{-1}\right) P_{\eta}
$$

In the case $n=m$ each representation in the decomposition of $\square^{\otimes N}$ has the same number of odd and even vectors, hence a zero super dimension. This actually becomes a vanishing super $q$ dimension. For any $X$ in $H_{N}, \operatorname{str} q^{H} X$ is thus equal to zero, and we cannot get a non-trivial link invariant by the method used for $n \neq m$. 


\section{Quantum $s l(1 / 1)$ and the Alexander-Conway Polynomial}

For simplicity we now discuss in more detail how the Conway-Alexander polynomial can be obtained from $U_{q} s l(1,1)^{1}$. The defining relations (1.12) read in this case

$$
\left[\eta, \eta^{+}\right]_{+}=\lambda \mathbf{1}, \quad \eta^{2}=\eta^{+2}=0,
$$

where the normalization factor $\lambda$ is at our disposal. If $\lambda=1$ in the fundamental representation, the coproduct formula (1.14) gives $\lambda=(N)_{q}$ in $\square^{\otimes N}$. We label the even state of $\square$ by 0 and the odd state by 1 . Then in 0,1 basis the fermion number operator is

$$
F=\left(\begin{array}{ll}
0 & 0 \\
0 & 1
\end{array}\right)
$$

In $\square^{\otimes N}, F=\sum_{a=1}^{N} F_{a}$, where $F_{a}$ acts as (2.2) on the $a^{\text {th }}$ representation and 1 otherwise.

In $\square$ we have

$$
\eta=\left(\begin{array}{ll}
0 & 1 \\
0 & 0
\end{array}\right), \quad \eta^{+}=\left(\begin{array}{ll}
0 & 0 \\
1 & 0
\end{array}\right)
$$

with $F=\eta^{+} \eta$. Setting $\lambda=1$ in (2.1) we then have for $\square^{\otimes N}$,

$$
\begin{gathered}
\eta=\frac{q^{-(N-1) / 2}}{(N)_{q}^{1 / 2}} \sum_{a=1}^{N-1} q^{a-1} \eta_{a}, \\
\eta^{+}=\frac{q^{-(N-1) / 2}}{(N)_{q}^{1 / 2}} \sum_{a=1}^{N-1} q^{a-1} \eta_{a}^{+},
\end{gathered}
$$

where $\eta_{a}=1 \otimes 1 \ldots \otimes \eta \otimes 1 \ldots$ Notice that $\eta$ being odd, $\eta_{a}$ does not act trivially on the representations other than $a^{\text {th }}$. It is possible to forget about the grading of the tensor product by adding strings of operators in (2.4) that reproduce the proper signs in commutation of odd objects. Equation (2.4) reads then ${ }^{2}$

$$
\begin{gathered}
\eta=\frac{q^{-(N-1) / 2}}{(N)_{q}^{1 / 2}} \sum_{b=1}^{N-1} q^{b-1} \Pi_{a<b}(-)^{F_{a}} \eta_{b}, \\
\eta^{+}=\frac{q^{-(N-1) / 2}}{(N)_{q}^{1 / 2}} \sum_{b=1}^{N-1} q^{b-1} \Pi_{a<b}(-)^{F_{a}} \eta_{b}^{+},
\end{gathered}
$$

where now $\eta_{b}$ acts as $\eta$ in $b^{\text {th }}$ representation and identity otherwise.

For any $X$ in $H_{N}$

$$
\begin{aligned}
& {[\eta, X]=0, \quad\left[\eta^{+}, X\right]=0,} \\
& {[F, X]=0 .}
\end{aligned}
$$

We refer to $\eta, \eta^{+}$as zero mode annihilation and creation operators. Finally we notice that

$$
\operatorname{str} X=\operatorname{Tr}(-)^{F} X .
$$

${ }_{1}^{1}$ It would be more correct to call the algebra $U_{q} g l(1,1)$ in this balanced case

2 Throughout this paper $(-)^{F}$ stand for $(-1)^{F}$ 
Any operator $X$ in $H_{N}$ has eigenvalues twice degenerate due to (2.6). These occur once in the sector with $F$ odd, once in the sector with $F$ even. Hence $\operatorname{str} X=0$.

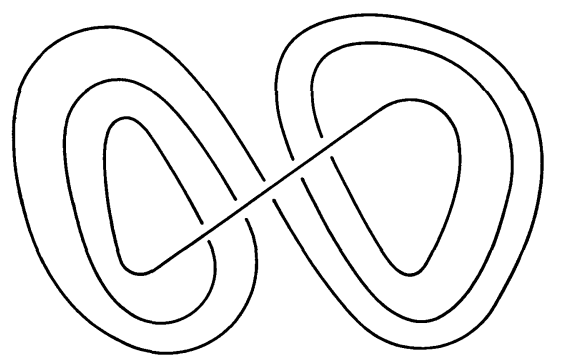

Fig. 2. $N=6$

A first way to get the Conway polynomial is - inspired by physics - to subtract the zero mode. We hence define for the link $\bar{B}$ closure of the braid $B$ on $N$ strings

$$
\nabla_{\bar{B}}\left(z=q-q^{-1}\right)=\frac{\operatorname{str} r_{N}(B) \eta \eta^{+}}{\operatorname{str} r_{1} \ldots r_{N-1} \eta \eta^{+}}
$$

where $B$ is some word on the $\sigma$ 's and $r_{a}$ acts as (1.19) on the $a^{\text {th }}$ and $a+1^{\text {th }}$ representations, and 1 otherwise.

A way of proving that $\nabla_{\bar{B}}(2.8)$ equals the Alexander-Conway polynomial of $\bar{B}$ is to compute it in the $H_{N}$ algebra in a recursive way that parallels the usual geometrical calculation based on Conway axioms. We notice that $\nabla$ vanishes for any braid corresponding to a split link since $\operatorname{str} X Y \eta \eta^{+}=0$ when $X, Y$ have no spaces in common onto which they act non-trivially. The numerator of (2.8) can then be rewritten using $(1.21,1.22)$ as a polynomial in $z$ times $\operatorname{str} r_{1} \ldots r_{N-1} \eta \eta^{+}$. Equation (1.21) in particular acts like the skein relation in Conway axioms. Finally the denominator of (2.8) ensures that $\nabla$ is 1 for the unknot, which is represented on $N$ strings as the closure of $\sigma_{1} \sigma_{2} \ldots \sigma_{N-1}$ (Fig. 2).

One can also show that the right-hand side of (2.8) actually enjoys the properties of a Markov trace with $\tau=\bar{\tau}=1$, thanks in particular to commutation of $\eta, \eta^{+}$with $H_{N}$. Since the correct skein relation holds, $\nabla$ coincides with the Alexander-Conway polynomial.

We notice for further purposes

$$
\operatorname{str} r_{1} \ldots r_{N-1} \eta \eta^{+}=(N)_{q}
$$

Example. Consider the trefoil knot represented by $B=\sigma^{3}-\ln \square^{\otimes 2}$. We have

$$
\begin{gathered}
\eta=\frac{q^{-1 / 2}}{\left(q+q^{-1}\right)^{1 / 2}}\left(\begin{array}{cccc}
0 & q & 1 & 0 \\
0 & 0 & 0 & 1 \\
0 & 0 & 0 & -q \\
0 & 0 & 0 & 0
\end{array}\right), \\
\eta^{+}=\frac{q^{-1 / 2}}{\left(q+q^{-1}\right)^{1 / 2}}\left(\begin{array}{rrrr}
0 & 0 & 0 & 0 \\
q & 0 & 0 & 0 \\
1 & 0 & 0 & 0 \\
0 & 1 & -q & 0
\end{array}\right),
\end{gathered}
$$




$$
\begin{gathered}
r \eta \eta^{+}=\frac{q^{-1}}{q+q^{-1}}\left(\begin{array}{ccrc}
q^{3}+q & 0 & 0 & 0 \\
0 & -q^{-1} & 1 & 0 \\
0 & 1 & -q & 0 \\
0 & 0 & 0 & 0
\end{array}\right), \\
r^{3} \eta \eta^{+}=\frac{q^{-1}}{q+q^{-1}}\left(\begin{array}{cccc}
q^{5}+q^{3} & 0 & 0 & 0 \\
0 & -q^{-3} & q^{-2} & 0 \\
0 & q^{-2} & -q^{-1} & 0 \\
0 & 0 & 0 & 0
\end{array}\right), \\
\operatorname{str} r \eta \eta^{+}=q+q^{-1}, \\
\operatorname{str} r^{3} \eta \eta^{+}=q^{3}+q^{-3} .
\end{gathered}
$$

Hence

$$
\nabla_{T}=\frac{q^{3}+q^{-3}}{q+q^{-1}}=1+\left(q-q^{-1}\right)^{2} .
$$

Using (2.1) we have as well

$$
\nabla_{\bar{B}}(z)=\frac{\operatorname{str} r_{N}(B) \eta^{+} \eta}{\operatorname{str} r_{1} \ldots r_{N-1} \eta^{+} \eta} .
$$

We shall come back to the interpretation of $\eta$ as a zero mode.

Besides (2.8) a more standard way to get the Alexander-Conway polynomial is to proceed via tangles

$$
\nabla_{\bar{B}}=\frac{\operatorname{str} r_{N}(B) \eta_{N} \eta_{N}^{+}}{\operatorname{str} r_{1} \ldots r_{N-1} \eta_{N} \eta_{N}^{+}}=\frac{-\operatorname{str} r_{N}(B) \eta_{N}^{+} \eta_{N}}{-\operatorname{str} r_{1} \ldots r_{N-1} \eta_{N}^{+} \eta_{N}}
$$

$\eta_{N} \eta_{N}^{+}$(respectively $\eta_{N}^{+} \eta_{N}$ ) enforces the last representation of $\square^{\otimes N}$ to carry 0 (respectively 1) state only.

Example. For the trefoil one has

$$
\begin{aligned}
\operatorname{str} r^{3} \eta_{N} \eta_{N}^{+} & =q^{3}-q+q^{-1}, \\
\operatorname{str} r \eta_{N} \eta_{N}^{+} & =q,
\end{aligned}
$$

and

$$
\nabla=q^{2}-1+q^{-2}=1+z^{2} .
$$

We notice the normalisations

$$
\begin{aligned}
& \operatorname{str} r_{1} \ldots r_{N-1} \eta_{N} \eta_{N}^{+}=q^{N-1} \\
& \operatorname{str} \eta_{1} \eta_{1}^{+} r_{1} \ldots r_{N-1}=q^{-(N-1)}
\end{aligned}
$$

\section{State Model}

We now come to the state model for braids. While (2.8), (2.11) look like a "Hamiltonian" formulation since a str is taken, it is easy to make a "Lagrangian" 
version by expanding terms in the trace. We thus define on the two-dimensional four-valent graph obtained by projection of the tangle a statistical mechanics model whose degrees of freedom are associated to edges and take values 0 or 1 [LW71]. We refer to 0 as the bosonic state and to 1 as the fermionic state. The interaction occurs at vertices with the following Boltzmann weights:

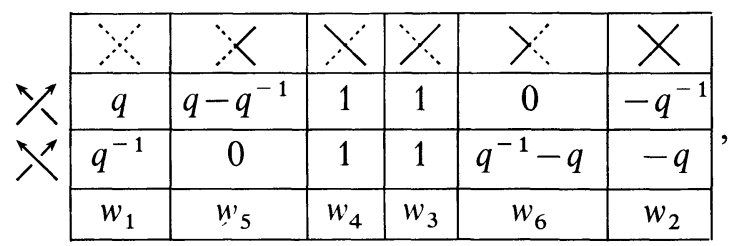

where a bosonic (fermionic) state is represented as a dashed (full) bond. Dashed (full) bonds draw possibly self-intersecting non-contractible loops on the graph. The Conway polynomial reads

$$
\nabla_{\bar{B}}=q^{-(N-1)} Z_{\text {loop }},
$$

where

$$
Z_{\text {loop }}=\sum_{\substack{\text { loop configurations } \\ \text { last strand carrying } \\ \text { bosonic or fermionic state }}}(-)^{\text {fermionic loop }} \prod_{\text {crossings }} \text { Boltzmann weights }
$$

and a fermion loop is a loop made of fermionic (full) bonds.

Example. Consider once again the trefoil

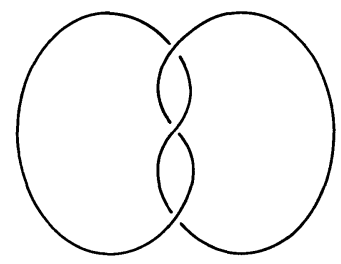

Loop configurations last strand being bosonic:
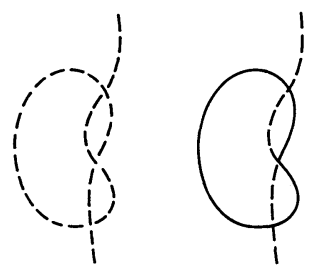

$$
\begin{array}{ccc}
B_{\text {weight }} & q^{3} & q-q^{-1} \\
(-)^{F} & 1 & -1 \\
Z_{\text {loop }}=q^{3}-q+q^{-1} \\
\nabla_{T}=q^{2}-1+q^{-2}=1+z^{2}
\end{array}
$$


Loop configuration last strand being fermionic:
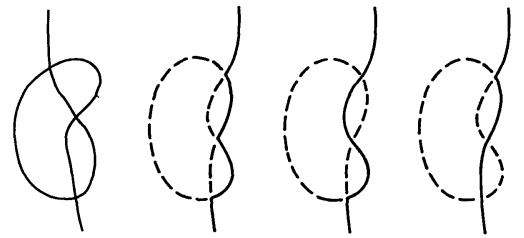

$$
\begin{aligned}
& B_{\text {weight }}-q^{-3} \quad\left(q-q^{-1}\right)^{3} \quad q-q^{-1} \quad q-q^{-1}
\end{aligned}
$$

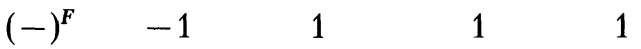

$$
\begin{aligned}
& Z_{\text {loop }}=\left(q-q^{-1}\right)^{3}+2\left(q-q^{-1}\right)+q^{-3}=q^{3}-q+q^{-1} \text {, } \\
& \nabla_{T}=1+z^{2} \text {. }
\end{aligned}
$$

Define the rotation number $\operatorname{rot}(\bar{B})$ as the sum of rotation numbers of all Seifert circuits in the decomposition of $\bar{B}$ (last strand being open). Rotation numbers for elementary circuits are shown below.

$$
\operatorname{rot}=+1 \bigcirc, \quad \operatorname{rot}=-1 \bigcirc
$$

The link diagram is decomposed into Seifert circuits by replacing each crossing by oriented parallel arcs as in $\lambda \mapsto=$

Example.

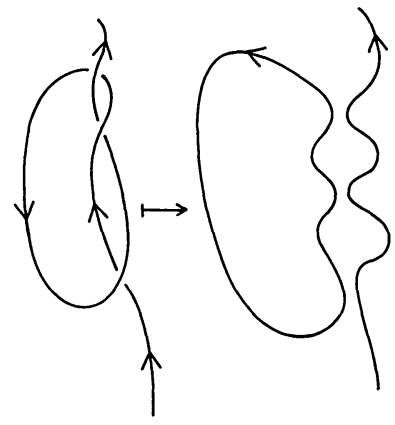

$$
\text { rot }=1
$$

Then (3.1) reads

$$
\nabla_{\bar{B}}=q^{-\operatorname{rot}(\bar{B})} Z_{\text {loop }}
$$

In fact a version of this model for arbitrary link diagrams appears in [K2, 89, $\mathrm{Ja} 87]$. What is called here bosonic (respectively fermionic) loop corresponds in $[\mathrm{K} 2,89]$ to a loop carrying label 1 (respectively -1 ). $q$ was called $t$ in $[\mathrm{K} 2,89]$. Finally the reader may notice some sign differences in $q$ exponents with $[K 2,89]$. They just occur from different convention in writing the $\check{R}$ matrix (compare (1.20) with the diagrammatics in $[\mathrm{K} 2,89])$.

Using proofs of $[K 2,89]$ we therefore know that formula (3.4) generalizes to an arbitrary tangle diagram.

We shall not comment more on state model details here but will take them up in a sequel to this paper (Jaeger, Kauffman, Saleur in preparation). Nevertheless, 
using for a short while notations of $[\mathrm{K} 2,89]$, it is worth mentioning that the basic form of these models is that of a partition function $\langle K\rangle=\sum_{\sigma}\langle K \mid \sigma\rangle \lambda^{\|\sigma\|}$, where $\sigma$ runs over the states of the diagram, $\langle K \mid \sigma\rangle$ denotes the product of local vertex weights and $\|\sigma\|$ is a global state evaluation (involving the state labels, loop counts, etc.). The key difference between the Alexander-Conway polynomial and other models is that $\lambda$ is a scalar, and that the model must be restricted to a subset of states (e.g., by fixing the value of an edge).

\section{Quandles, Crystals and the Burau Representation}

As it happens, much of classical knot theory can be summarized by describing an attempt to generalize the idea of coloring the arcs of a link diagram to form a colored state whose properties are invariant under the Reidemeister moves. This colored method is elementary and shows that most of classical knot theory can be seen as statistical mechanics. By using this approach, we shall be able to make identifications between the two subjects from the very beginning. In particular we shall see that the classical Burau representation directly describes a free fermion. The simplest example of such a coloring is the coloring of the trefoil knot. In this

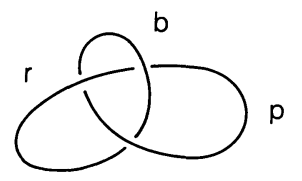

figure the representation shown for the trefoil is colored in three colors: red $(r)$, blue $(b)$, and purple $(p)$. The diagram is composed of three arcs - one of each color. Thus, we have a color space $\mathscr{C}(K)$ consisting of three elements. In order to see that this color space gives rise to a topological invariant for the trefoil, we adopt a rule of one or three: Any crossing must have either three distinct colors incident to it or only one color incident to it.

With this assumption, it is easy to see that a knot diagram (one component) that is tri-colored, can remain tri-colored under any Reidemeister move (via a local change in the coloring of the diagram). On the other hand, there is no way to induce a tri-coloration of an unknotted diagram-starting from the unknot and following these rules (i.e., making local changes at each move). Thus we conclude that the existence of a tri-coloration obeying the rule of one or three is a topological property of a knot diagram, and thus the trefoil knot is indeed knotted.

How can one generalize this coloring scheme? One direction is to assume that each arc of the diagram $K$ is labelled from elements of a set $\mathscr{C}(K)$ that we shall call the color space of $K$, and that there is a rule for composing colors so that the arcs incident to a crossing obey this rule. We shall assume that for colors $a, b \in \mathscr{C}(K)$ there is a third color $a \bar{b} \in \mathscr{C}(K)$ and also another third color $a \sqrt{b} \in \mathscr{C}(K)$. The notation $a \bar{b}$ connotes a non-commutative binary operation between $a$ and $b$ (it is associative), where $\bar{b}$ is a symbolic unary operation performed on $b$ making $b$ into an operator on the color space. That is, for each $b \in \mathscr{C}$ we have $\bar{b}, \bar{b}: \mathscr{C} \rightarrow \mathscr{C}$ and the evaluations $\vec{b}(\chi), \sqrt{b}(\chi)$ for $\chi \in \mathscr{C}$ are written

$$
\left\{\begin{array}{l}
\bar{b}(\chi)=\chi \bar{b} \\
\bar{b}(\chi)=\chi \sqrt{b} .
\end{array}\right.
$$


Associativity of the composition of functions makes this mixed algebra of operators and operands associative. We let $\widehat{\mathscr{C}}$ denote the set of compositions of operators derived from $\mathscr{C}$.

With $\mathscr{C}$ and $\mathscr{C}$ given we can define the local color rules:
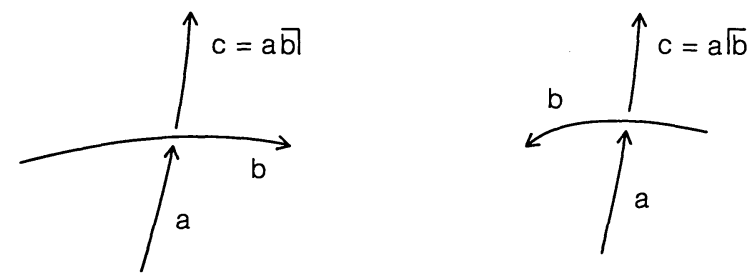

Then, we assign the color $a \bar{b}$ or $a \sqrt{b}$ (according to orientation) to the out-going arc at a crossing. Here $b$ is the over-crossing line and $a$ is the in-coming undercrossing arc. The operator $\bar{b}$ is assigned to a positive crossing, while the operator $\bar{b}$ is given to a negative crossing.

We now examine the properties of this operator algebra on the color space in regard to the Reidemeister moves. First consider move II:
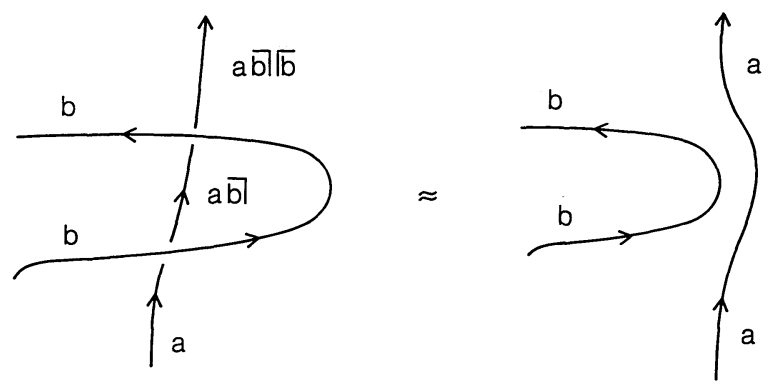

We see at once that $a \bar{b} \sqrt{b}=a$ for all $a$. Hence we must assume that

$$
\bar{b} \mid \bar{b}=1 \text { and } \quad \bar{b} b \bar{b}=1 \text {, }
$$

where 1 is the identity transformation of $\mathscr{C}$. This means that the set of operators $\mathscr{\mathscr { C }}$ forms a group.

Each crossing in the diagram $\mathscr{K}$ gives rise to a relation in the group $\mathscr{\mathscr { C }}$. In order to see this, consider the following version of the type III Reidemeister move:

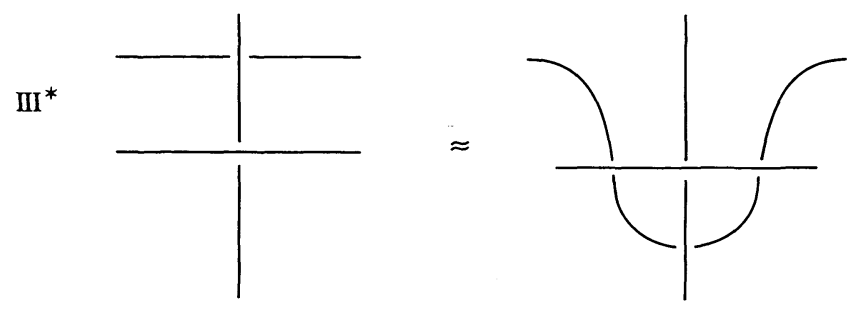

In this version of the type III move, the strand taking a journey through two regions is replaced locally by a journey through the other two regions.

The move III* can be accomplished via a combination of moves II and III. 
Indeed

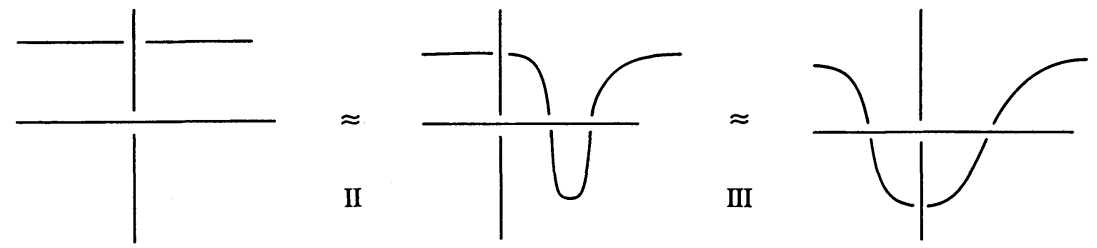

Thus, we can (in the presence of II) replace the move III by III*.

Now observe the effect of III* on the algebra of operators:
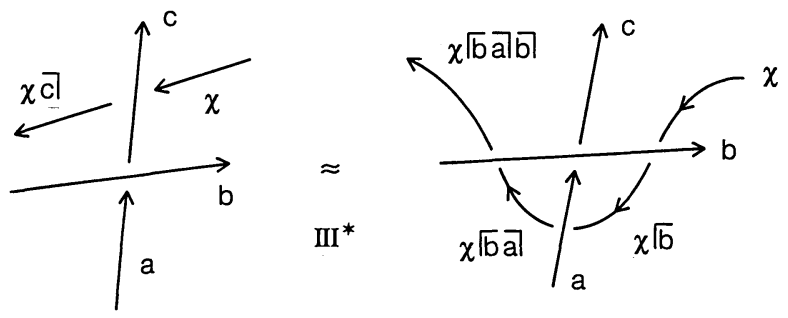

This diagram shows, that, to preserve coloring, we need to assume that

$$
\bar{c}=|\bar{b} a \bar{b}|
$$

whenever $c=a \bar{b}$.

In other words, as operators we have

$$
\bar{c}=\left.\bar{b}\right|^{-1} \bar{a} \bar{b}
$$

at the crossing.

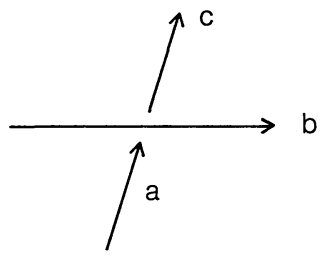

And $c=\sqrt{b}^{-1} \vec{a} \mid \sqrt{b}$ at the crossing of opposite type.

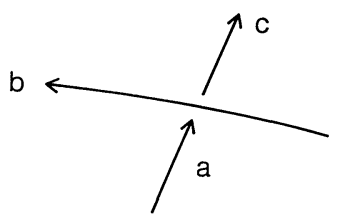

More generally, we must assume the rules

$$
\left\{\begin{array}{rl}
\overline{a \bar{b}} \mid & =\sqrt{b} \bar{a} \bar{b} \\
\mid \overline{a \bar{b}} & =\sqrt{b} \sqrt{a} \bar{b} \mid \\
\sqrt{a \sqrt{b}} & =\bar{b} \mid \sqrt{a} \sqrt{b} \\
\bar{a}|\bar{b}| & =\bar{b} \mid \bar{a} \bar{b}
\end{array} .\right.
$$

These rules describe how the operator algebra interacts with the color space. The pair $(\mathscr{C}, \widehat{\mathscr{C}})$ will be called the crystal $[\mathrm{K} 1,87]$ and [K91] of the knot or link K. It is a generalization of the fundamental group. In fact the group $\mathscr{\mathscr { C }}(K)$ is, by itself, isomorphic with the fundamental group of $K$. From the crystal one can derive the 
quandle of Joyce [JY82]. Hence it follows from Joyce's work that the crystal classifies unoriented knot types up to mirror images.

There are other possibilities for generalizing color states for knot and link diagrams, but the crystal/quandle/fundamental group is one generalization that makes direct contact with the classical knot theory.

In order to bring these relations down to earth, and in order to see how the Burau representation of the braid group makes its appearance in this theory, we now consider linear representations of the crystal operations. That is, let us assume that $a \bar{b} \mid$ and $a \sqrt{b}$ are given by linear formulas

$$
\begin{aligned}
a \bar{b} & =R a+S b, \\
a\lceil b & =R^{\prime} a+S^{\prime} b,
\end{aligned}
$$

where $R$ and $S$ are invertible elements in a commutative ring $\mathscr{R}$ and the color space $\mathscr{C}$ is a module over this ring $\mathscr{R}$.

In order for $a \bar{b} \mid$ and $a \sqrt{b}$ to satisfy the crystal relations for the Reidemeister moves II and III*, it is necessary that a linear representation (as described above) takes the form

$$
\begin{aligned}
a \bar{b} & =t a+(1-t) b, \\
a \sqrt{b} & =t^{-1} a+\left(1-t^{-1}\right) b,
\end{aligned}
$$

where $t$ is a unit in the ring $\mathscr{R}$.

Proof. Omitted. See [K91].

The universal version of the linear crystal representation in this proposition ensues when we take the ring $\mathscr{R}$ to be the integral group ring of the infinite cyclic group generated by the powers of a transcendental variable $t$. That is, we take $\mathscr{R}=\mathbf{Z}\left[t, t^{-1}\right]$, where $\mathbf{Z}$ denotes the integers. The elements of $\mathscr{R}$ are finite Laurent polynomials in $t$. Call this the Alexander Crystal of the link $K$.

It is useful to verify the crystal properties for this representation. Thus

$$
\begin{aligned}
a \bar{b} \mid \sqrt{b} & =(t a+(1-t) b) b \\
& =t^{-1}(t a+(1-t) b)+\left(1-t^{-1}\right) b \\
& =a+\left(t^{-1}-1\right) b+\left(1-t^{-1}\right) b \\
& =a, \\
\chi \overline{a \bar{b}} & =t \chi+(1-t) a \bar{b} \\
& =t \chi+(1-t)(t a+(1-t) b) \\
& =t \chi+\left(t-t^{2}\right) a+\left(1-2 t+t^{2}\right) b
\end{aligned}
$$

while

$$
\begin{aligned}
\chi\lceil\bar{b} \vec{a} \bar{b}] & =\left(t^{-1} \chi+\left(1-t^{-1}\right) b\right) \bar{a} \bar{b} \\
& =\left[t\left(t^{-1} \chi+\left(1-t^{-1}\right) b\right)+(1-t) a\right] \bar{b} \\
& =t\left[t\left(t^{-1} \chi+\left(1-t^{-1}\right) b\right)+(1-t) a\right]+(1-t) b \\
& =t \chi+t(t-1) b+\left(t-t^{2}\right) a+(1-t) b \\
& =t \chi+\left(t-t^{2}\right) a+\left(t^{2}-2 t+1\right) b .
\end{aligned}
$$

Thus $a \bar{b}|=| \bar{b} a \bar{b} \mid$. The other instances of III* follow similarly.

The module structure of $\mathscr{C}(K)$ over $\mathbf{Z}\left[t, t^{-1}\right]$ is called the Alexander module. It is generated by one symbol for each arc in the diagram $\mathscr{K}$, and one relation. 
Example. Consider the trefoil with relations in the Alexander module

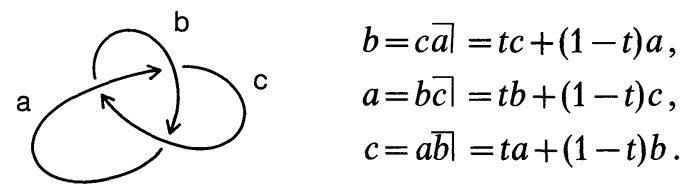

The relation matrix is

$$
M(K)=\begin{array}{c|c|c|c|} 
& a & b & c \\
\hline a & 1-t & -1 & t \\
\hline b & -1 & t & 1-t \\
\hline c & t & 1-t & -1 \\
\hline
\end{array} .
$$

Note that we have labelled the rows and columns of this matrix by the arcs in the diagram. (The arcs are in 1-1 correspondence with the crossings by associating the crossing at the base of an arc to the given arc.) Each row gives the coefficients of the relation corresponding to a given arc. Note that in this example the three rows are linearly dependent. This is generally the case, and leads to the definition of the Alexander polynomial as the determinant of a minor of $M(K)$ :

$$
\Delta_{\mathbf{K}}(t) \doteq \operatorname{det}^{\prime}(M(K)),
$$

where $\operatorname{det}^{\prime}(M)$ is the determinant of any $(N-1) \times(N-1)$ minor of $M(K)$, and $\doteq$ means equality up to multiplication by $\pm t^{i}(i \in \mathbf{Z})$. Thus we have, in the case of the trefoil

$$
\begin{aligned}
\Delta_{T}(t) & \doteq \operatorname{det}\left(\begin{array}{cc}
t & 1-t \\
1-t & -1
\end{array}\right) \\
& \doteq-t-\left(1-2 t+t^{2}\right), \\
\Delta_{T}(t) & \doteq-t^{2}+t-1 \doteq t^{2}-t+1 .
\end{aligned}
$$

The significance of the Alexander polynomial with respect to the color space $\mathscr{C}(K)$ lies in the fact that $\Delta_{K}(t)$ is a generator of the annihilator ideal of $\mathscr{C}(K)$ in $\mathbf{Z}\left[t, t^{-1}\right]$. This is, $\Delta_{K}(t) \cdot \chi=0$ for all $\chi \in \mathscr{C}(K)$, and $\Delta_{K}(t)$ generates the ideal of Laurent polynomials with this property. It is of interest to see how the Alexander polynomial arises directly in terms of this property. Take the case of the trefoil as shown above. If $a=0$ then $c=a \vec{b}=t a+(1-t) b=(1-t) b$. Hence $0=a=b \bar{c}=t b$ $+(1-t)^{2} b=\Delta_{K}(t) b$.

Since the Alexander polynomial is a determinant, it can be expanded combinatorially in various ways. Furthermore, there are various ways to obtain determinantal expressions for this polynomial. An expression that is very closely related to the Alexander module/matrix arises for braids in the form of the Burau representation of the Artin braid group. The Burau representation derives directly from the linear representation of the crystal as follows: Consider a crossing as shown below.

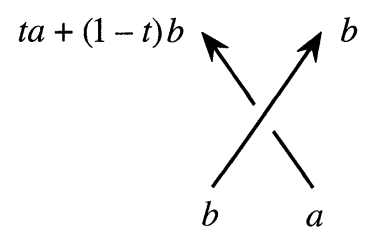


We have labelled the arcs incident to this crossing in accordance with the rule $a \bar{b}=c=t a+(1-t) b$. Now regard $a$ and $b$ as basis elements for a vector space $V$, and define $\varrho: V \rightarrow V$ by $(a) \varrho=b,(b) \varrho=t a+(1-t) b$. Thus, as a matrix with respect to the basis $\{b, a\}$, we have ( $\varrho$ acts on the left.)

$$
\varrho=\left(\begin{array}{cc}
1-t & t \\
1 & 0
\end{array}\right)
$$

For the inverse crossing we have $\varrho^{-1}=\left(\begin{array}{cc}1 & 0 \\ t^{-1} & 1-t^{-1}\end{array}\right)$. The matrices $\varrho$ and $\varrho^{-1}$ then form the building blocks for the Burau representations $\varrho_{N}: B_{N}$ $\rightarrow M_{N}\left(\mathrm{Z}\left[t, t^{-1}\right]\right)$, where $B_{N}$ denotes the $N$-strand braid group and $M_{N}\left(\mathbf{Z}\left[t, t^{-1}\right]\right)$ denotes the ring of $N \times N$ matrices over $\mathbf{Z}\left[t, t^{-1}\right]$.

Letting $V_{N}$ denote the free module over $\mathbf{Z}\left[t, t^{-1}\right]$ with basis $\left\{v_{1}, v_{2}, \ldots, v_{N}\right\}\left(v_{a}\right.$ corresponds to the $a^{\text {th }}$ braid strand), we let $\varrho_{N}: B_{N} \rightarrow \operatorname{Aut}\left(V_{N}\right)$ denote the Burau Representation:

$$
v_{a} \varrho_{N}\left(\sigma_{b}\right)= \begin{cases}(1-t) v_{b}+t v_{b+1}, & a=b \\ v_{b} & a=b+1 . \\ v_{a} & \text { otherwise }\end{cases}
$$

In other words, $\varrho_{N}\left(\sigma_{a}\right)$ is the Burau matrix $\sigma$ on the subspace $\left\{v_{a}, v_{a+1}\right\}$ and the identity elsewhere.

It is easy to see that $\varrho_{N}$ is a representation of the braid group: The braid group has relations $\sigma_{a} \sigma_{a+1} \sigma_{a}=\sigma_{a+1} \sigma_{a} \sigma_{a+1}, \sigma_{a} \sigma_{a}^{-1}=1$ and $\sigma_{a} \sigma_{b}=\sigma_{b} \sigma_{a}(|a-b| \geqq 2)$. These correspond to type III, type II and background Reidemeister moves. Hence our work in verifying the linear representation of the Crystal translates directly into a proof of the well-definedness of the Burau representation. With the Burau representation in hand, we can return to the color space and Alexander module $\mathscr{C}(\bar{B})$ for the closure of a braid word $B \in B_{N}$. The basic fact is that if $B \in B_{N}$ then $\mathbf{M}(B)$ $=\varrho_{N}(B)-I$ is a relation matrix for the Alexander module (color space) $\mathscr{C}(\bar{B})$. Furthermore, the Alexander polynomial is given by the formula

$$
\Delta_{\bar{B}}(t) \doteq \operatorname{det}^{\prime}(\mathbf{M}(B)),
$$

where $\operatorname{det}^{\prime}$ denotes the determinant of any $(N-1) \times(N-1)$ minor of $\mathbf{M}(B)$. (See [B74] for proofs of these facts.)

Example.

$$
\begin{aligned}
& \varrho(B)=\left(\begin{array}{cc}
1-t & t \\
1 & 0
\end{array}\right)^{3} \\
& B=\sigma_{1}^{3} \\
& \bar{B}=T
\end{aligned}
$$

Thus $\operatorname{det}^{\prime} \mathbf{M}(B) \doteq\left(1+t-t^{2}\right)$ and $\Delta_{\bar{B}}(t) \doteq t^{2}-t+1$. 
This last model of the Alexander polynomial is the one that translates directly into the $U_{q} s l(1,1)$ state model (Sect. 3) via the formula $\operatorname{det}(A+s I)=\operatorname{tr}(\mathscr{S} \widehat{A})$ (Appendix). We shall discuss this translation in the next section (Sect. 5). For now it is appropriate to return to our original version of the Alexander matrix for an arbitrary link diagram, and to point out some further connections both with state models, and with some classical aspects of knot theory. First of all, the same formula $\operatorname{det}(A+S I)=\operatorname{tr}(\mathscr{S} \hat{A})$ applies to the first Alexander matrix. We see that $M(K)=-I+N(K)$, where $N(K)$ is the matrix of relations at the base of each arc in the diagram. For example, in the case of the trefoil, we find

$$
\mathbf{M}(K)=\left[\begin{array}{rrr}
-1 & 0 & 0 \\
0 & -1 & 0 \\
0 & 0 & -1
\end{array}\right]+\left[\begin{array}{ccc}
1-t & 0 & t \\
0 & t & 1-t \\
t & 1-t & 0
\end{array}\right]
$$

This gives yet another expression for the Alexander polynomial as a super-trace that is apparently different from our other models.

Finally, it is worth pointing out that the crystal version of the Alexander matrix is identical to that obtained using standard techniques on the fundamental group of the knot complement. These standard techniques involve the free differential calculus of Fox [F63]. We give a quick sketch of this relationship below, because it is significant that at least in the case of the Alexander polynomial, one can derive a solution to the Yang-Baxter equation from the fundamental group of the knot complement. Let $G$ be a group (written multiplicatively). Let $\Gamma=\mathbf{Z}[G]$ denote the group ring of $G$. That is, $\Gamma$ consists of all formal sums $n_{1} g_{1}+n_{2} g_{2}+\ldots+n_{k} g_{k}$, where $n_{i} \in \mathbf{Z}$ is an integer and $g_{i}$ is a member of $G$. A (Fox) derivation $D: \Gamma \rightarrow \Gamma$ is a linear mapping that satisfies the formula $D(g h)=D(g)+g D(h)$ for $g, h \in \Gamma$.

This notion of derivation is motivated by the fact that the formula for lifting elements of $G=\pi_{1}(X)\left(\pi_{1}\right.$ denotes the fundamental group of a space $\left.X\right)$ into the universal covering $\tilde{X}$ is given by $\widetilde{g h}=(\tilde{g})+g(\tilde{h})$. Here $\tilde{g h}, \tilde{g}, \tilde{h}$ denote path lifts, and the multiplication by $g$ connotes the action of $G=\pi_{1}(X)$ on the space $\tilde{X}$.

Given a presentation of a group $G=\left(\chi_{1}, \chi_{2}, \ldots, \chi_{n} / r_{1}, r_{2}, \ldots, r_{m}\right)$ we can form the Jacobian matrix

$$
\mathrm{jac}=\left(\frac{\partial r_{i}}{\partial \chi_{j}}\right)
$$

where $\frac{\partial}{\partial \chi_{j}}$ denote derivations of this kind, and

$$
\frac{\partial \chi_{i}}{\partial \chi_{j}}=\delta_{i j}=\left\{\begin{array}{ll}
1 & i=j \\
0 & i \neq j
\end{array} .\right.
$$

If $G$ is the Wirtinger presentation of the knot group then we can take $m=n$ and there is a homomorphism $\phi: \mathbf{Z}[G] \rightarrow \mathbf{Z}\left[t, t^{-1}\right]$ naturally associated with $G$. Fox proved [F63] that the Alexander polynomial was given by a formula: $\Delta_{K}(t) \doteq \operatorname{det}^{\prime}\left(\mathrm{jac}^{\phi}\right)$, where jac denotes a Jacobian matrix for a presentation of the fundamental group of $K, \phi$ takes the entries of jac to $\mathbf{Z}\left[t, t^{-1}\right]$ and det' denotes the determinant of any $(N-1) \times(N-1)$ minor of $\mathrm{jac}^{\phi}$.

In fact, for the Wirtinger presentation the Jacobian matrix $\mathrm{jac}^{\phi}$ is exactly our first matrix of relations $\mathbf{M}(\mathbf{K})$. It is illuminating to see how this comes about:

0 . The Wirtinger presentation for the fundamental group of the knot complement is obtained from the crystal by replacing $a \bar{b} \mid$ by $b^{-1} a b$ and $a \sqrt{b}$ by $b a b^{-1}$. 
1. Note that if $D: \Gamma \rightarrow \Gamma$ is a Fox derivation, then

$$
D\left(g^{-1}\right)=-g^{-1} D(g) \quad\left(0=D\left(g g^{-1}\right)=D(g)+g D\left(g^{-1}\right)\right) .
$$

2. Let $a \bar{b}=b^{-1} a b, a \sqrt{b}=b a b^{-1}$, where $a, b \in G$ a given group. Then

$$
\begin{aligned}
D(a \bar{b}) & =-b^{-1} D(b)+b^{-1}(D(a)+a D(b)) \\
& =b^{-1} D(a)+\left(b^{-1} a-b^{-1}\right) D(b)
\end{aligned}
$$

and

$$
\begin{aligned}
D(a \sqrt{b}) & =D(b)+b\left(D(a)+a\left(-b^{-1} D(b)\right)\right) \\
& =b D(a)+\left(1-a b^{-1}\right) D(b) .
\end{aligned}
$$

3. Thus if $\phi(a)=\phi(b)=t^{-1}$ and $\mathbf{D}=\phi D$, then

$$
\begin{aligned}
& \mathbf{D}(a \bar{b})=t \mathbf{D}(a)+(1-t) \mathbf{D}(b) \\
& \mathbf{D}(a \sqrt{b})=t^{-1} \mathbf{D}(a)+\left(1-t^{-1}\right) \mathbf{D}(b) .
\end{aligned}
$$

It is in exactly this way the Fox calculus produces the Alexander module and basic crystal representation from the fundamental group of the complement of the knot. This relationship is of interest in the context of the $U_{q} s l(1,1)$ model of the Alexander polynomial since it shows how the vertex weights for a solution to the Yang-Baxter equation can arise from the combinatorial group theory of the knot group. We expect deeper connections with the classical topology to emerge in this line.

\section{From the Burau Representation to Quantum $s l(1 / 1)$}

The boson-fermion approach we used so far can be formulated in an interesting way using exterior products. To each basic state of $\square^{\otimes N}$ we associate in $\Lambda^{k}$ a formal product $v_{a_{1}} \wedge \ldots \wedge v_{a_{k}}$ of basic elements $v_{1} \ldots v_{N}$ with the rule

$$
\begin{gathered}
(0 \ldots 010 \ldots 010 \ldots 01 \ldots) \\
a_{1} \ldots a_{i} \ldots a_{k}
\end{gathered} \rightarrow v_{a_{1}} \wedge \ldots \wedge v_{a_{k}},
$$

where $a$ 's label the positions of elementary fermions. We may think as well of 1 as $(0 \ldots 0) \equiv \Omega$ or as a ground state and of $v_{a_{1}} \wedge v_{a_{2}} \wedge \ldots \wedge v_{a_{k}}$ as $\eta_{a_{1}}^{+} \eta_{a_{2}}^{+} \ldots \eta_{a_{k}}^{+} \Omega$. Wedge products are associative, anticommutative, and linear. The exterior algebra associated with $\square^{\otimes N}$ is $\wedge^{*}=\wedge^{0} \oplus \wedge^{1} \oplus \ldots \oplus \wedge^{N}$.

Now consider some operator in $\square^{\otimes 2}$ conserving $F$

$$
\hat{X}=\left(\begin{array}{cccc}
1 & 0 & 0 & 0 \\
0 & w_{5} / w_{1} & w_{3} / w_{1} & 0 \\
0 & w_{4} / w_{1} & w_{6} / w_{1} & 0 \\
0 & 0 & 0 & w_{2} / w_{1}
\end{array}\right) .
$$

Basis states read in the wedge product language $(00) \rightarrow 1,(01) \rightarrow v_{2},(10) \rightarrow v_{1},(11)$ $\rightarrow v_{1} \wedge v_{2}$. If the weights (5.2) satisfy moreover the "free fermion" relation (as (3.1))

$$
w_{1} w_{2}+w_{3} w_{4}=w_{5} w_{6}
$$


it is possible to express $\hat{X}$ in terms of another operator $X$ that acts on one fermion states only. Indeed set in $v_{2}, v_{1}$ basis

$$
X=\left(\begin{array}{ll}
w_{5} / w_{1} & w_{3} / w_{1} \\
w_{4} / w_{1} & w_{6} / w_{1}
\end{array}\right)
$$

and lift the action of $X$ from $\wedge^{1}$ to $\wedge^{*}$ by

$$
\begin{aligned}
1 \hat{X} & =1 \\
\left(v_{1} \wedge v_{2}\right) \hat{X} & =v_{1} X \wedge v_{2} X \\
& =\frac{w_{5} w_{6}-w_{3} w_{4}}{w_{1}^{2}} v_{1} \wedge v_{2} .
\end{aligned}
$$

$\hat{X}$ coincides with (5.2) due to (5.3). The "free fermion" name of (5.3) stems from the fact that action of $\hat{X}$ on multifermion states can be factorized onto its action on one fermion states only without multiparticle interaction terms. We shall come back to this in Sect. 6.

In general for any operator $X$ defined by its action on basic states $v_{1} \ldots v_{N}$ of $\wedge^{1}$ we introduce the lifted operator $\hat{X}$ in $\wedge^{*}$ by

$$
\left(v_{a_{1}} \wedge \ldots \wedge v_{a_{k}}\right) \hat{X}=v_{a_{1}} X \wedge \ldots \wedge v_{a_{k}} X
$$

We introduce also the free fermion number operator

$$
\left(v_{a_{1}} \wedge \ldots \wedge v_{a_{k}}\right) F=k
$$

(of course $(-)^{F}$ is the simplest example of a free fermion operator). Then a useful result is

$$
\begin{aligned}
\operatorname{det}(X-I) & =(-)^{N} \operatorname{tr}(-)^{F} \hat{X} \\
& =(-)^{N} \operatorname{str} \hat{X}
\end{aligned}
$$

which connects the determinant of a $N \times N$ matrix to the supertrace of a $2^{N} \times 2^{N}$ one. This is proven in the Appendix.

We can now show how the $R$-matrix for the Alexander-Conway polynomial (as described in Sect. 2) arises from the linear crystal representation by going to the exterior algebra: We have

$$
\begin{gathered}
1 \hat{\varrho}, \quad a \hat{\varrho}=b, \quad b \hat{\varrho}=t a+(1-t) b, \\
(a \wedge b) \hat{\varrho}=(a \varrho) \wedge(b \varrho)=-t(a \wedge b) .
\end{gathered}
$$

Hence for the small exterior algebra basis we have

$$
\hat{\varrho}=\left(\begin{array}{cccc}
1 & 0 & 0 & 0 \\
0 & 1-t & t & 0 \\
0 & 1 & 0 & 0 \\
0 & 0 & 0 & -t
\end{array}\right) .
$$

The basis ordering $\{1, b, a, a \wedge b\}$ corresponds to the coding adapted above

$$
1 \leftrightarrow 00, \quad b \leftrightarrow 01, \quad a \leftrightarrow 10, \quad a \wedge b \leftrightarrow 11 .
$$


On the other hand

$$
q^{-1} \check{R}=\left(\begin{array}{cccc}
1 & 0 & 0 & 0 \\
0 & 1-q^{-2} & q^{-1} & 0 \\
0 & q^{-1} & 0 & 0 \\
0 & 0 & 0 & -q^{-2}
\end{array}\right) .
$$

Hence $q^{-1} \breve{R}$ can be identified with $\varrho$ after the renormalizations $t \rightarrow q^{-2}$,

$$
v_{2} \rightarrow q^{1 / 2}(01), \quad v_{1} \rightarrow q^{-1 / 2}(10) .
$$

Such a change of basis does not affect the supertrace, allowing us to write

$$
\operatorname{det}\left(\varrho_{N}(B)-I\right)=(-)^{N} q^{-e(B)} \operatorname{str} r_{N}(B) .
$$

Both sides of (5.11) are actually zero. We met this difficulty for the str in Sect. 2. As far as the det is concerned, it vanishes because $\varrho(B)$ leaves the vector $v_{1}+\ldots+v_{N}$ with all coordinates equal invariant.

An easy way to regularize the determinant is to suppress the zero mode. If $\lambda_{1} \ldots \lambda_{N}$ are the eigenvalues of $\varrho(B)$ with $\lambda_{1}=1$ we define

$$
\operatorname{det}^{\prime}(\varrho(B)-I) \stackrel{\text { def }}{\equiv} \prod_{a=2}^{N}\left(\lambda_{a}-1\right)
$$

which equals the sum of diagonal minors of $\varrho(B)-I$. Writing

$$
\wedge^{1}=\operatorname{ker}(\varrho(B)-I) \oplus \wedge^{1^{\prime}},
$$

$\operatorname{det}^{\prime}$ is the determinant of the operator $\varrho(B)-I$ restricted to $\wedge^{1^{\prime}}$.

After lifting to $\wedge^{*}$, the existence of $\lambda_{1}=1$ translates into the double degeneracy of $r(B)$ eigenvalues. Introducing the linear operator in $\wedge^{*}$,

$$
Z^{+}: v_{a_{1}} \wedge \ldots \wedge v_{a_{k}} \rightarrow\left(v_{1}+\ldots+v_{N}\right) \wedge\left(v_{a_{1}} \wedge \ldots \wedge v_{a_{k}}\right),
$$

and writing

$$
\wedge^{*}=\operatorname{ker} Z^{+} \oplus \wedge^{* \prime},
$$

Eq. (5.8) becomes

$$
\operatorname{det}^{\prime}\left(\varrho_{N}(B)-I\right)=(-)^{N-1} q^{-e(B)} \operatorname{str}_{\wedge *} r_{N}(B) .
$$

We introduce

$$
Z: v_{a_{1}} \wedge \ldots \wedge v_{a_{k}} \rightarrow \sum_{a_{k}=a_{1}}^{a_{k}} v_{a_{1}} \wedge \ldots \wedge v_{a_{k}^{\prime}}^{0} \wedge \ldots \wedge v_{a_{k}}(-)^{k^{\prime}-1},
$$

where $\stackrel{0}{v}$ means the vector is omitted in the wedge product.

One checks

$$
\left[Z, Z^{+}\right]_{+}=N, \quad Z^{2}=Z^{+2}=0 .
$$

The projector on $\wedge^{* \prime}$ is $\frac{Z Z^{+}}{N}$, hence (5.14) reads

$$
\operatorname{det}^{\prime}\left(\varrho_{N}(B)-I\right)=(-)^{N-1} q^{-e(B)} \operatorname{str}\left(r_{N}(B) \frac{Z Z^{+}}{N}\right) .
$$

Performing the right change of basis such that $\varrho_{N} \widehat{(B)}$ becomes $q^{-e(B)} r_{N}(B)$, we see that the normalized $Z, Z^{+}$operators become identical to the $\eta, \eta^{+}$zero mode 
operators introduced in Sect. 2. For the unknot (5.17) reads

$$
\operatorname{det}^{\prime}\left(\varrho_{1} \ldots \varrho_{N-1}-I\right)=(-)^{N-1} q^{-(N-1)} \operatorname{str}\left(r_{1} \ldots r_{N-1} \eta \eta^{+}\right)
$$

and

$$
\nabla_{\bar{B}}=(-)^{N-1} q^{e(B)} \frac{\operatorname{det}^{\prime}\left(\varrho_{N}(B)-I\right)}{(N)_{q}}
$$

in agreement with [Jo87].

Example. For the trefoil

$$
\begin{gathered}
\varrho(B)=\left(\begin{array}{cc}
1-q^{-2}+q^{-4}-q^{-6} & q^{-2}-q^{-4}+q^{-6} \\
1-q^{-2}+q^{-4} & q^{-2}-q^{-4}
\end{array}\right) \\
\operatorname{det}^{\prime}(\varrho(B)-I)=\sum \text { diag. minors }=-q^{-6}-1 \\
\nabla_{\text {Trefoil }}=-q^{3} \frac{-q^{-6}-1}{q+q^{-1}}=\frac{q^{3}+q^{-3}}{q+q^{-1}}=1+z^{2}
\end{gathered}
$$

For the link

$$
\begin{aligned}
\varrho & =\left(\begin{array}{cc}
1-q^{-2}+q^{-4} & q^{-2}-q^{-4} \\
1-q^{-2} & q^{-2}
\end{array}\right), \\
\operatorname{det}^{\prime}(\varrho-I) & =q^{-4}-1, \\
\nabla & =-q^{2} \frac{q^{-4}-1}{q+q^{-1}}=q-q^{-1}=z .
\end{aligned}
$$

Instead of det' defined by (5.12) we may focus on say the $N^{\text {th }}$ diagonal minor of $\varrho(B)-I$. Then by an easy generalization of the above

$$
\Delta_{11}\left(\varrho_{N}(B)-I\right)=(-)^{N-1} \operatorname{str} q^{-\varrho(B)} r_{N}(B) \eta_{N} \eta_{N}^{+}
$$

and from (2.12),

$$
\nabla_{\bar{B}}=(-)^{N-1} q^{e(B)-N+1} \Delta_{11}\left(\varrho_{N}(B)-I\right)
$$

\section{Free Fermion Description}

We now come to a reformulation of the state model where the tangle diagram will play the role of a Feynman graph for a fermionic theory.

To each crossing $i$ of an oriented tangle diagram we associate 4 Grassmann variables $\psi_{i, u} ; \psi_{i, u}^{+} ; \psi_{i, d} ; \psi_{i, d}^{+}$. The labels $u$ and $d$ refer to edges going up and down with respect to the direction of the crossing as shown below.

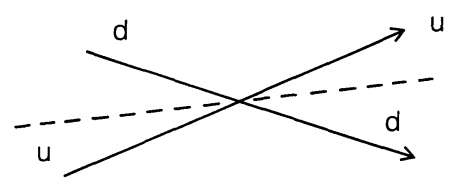


All $\psi$ 's anticommute

$$
\psi_{i, \alpha}^{x} \psi_{j, \beta}^{y}=-\psi_{j, \beta}^{y} \psi_{i, \alpha}^{x}, \quad \alpha, \beta=u \quad \text { or } \quad d \quad x, y=\text { none or }+.
$$

In particular a $\psi$ squared is always zero,

$$
\left(\psi_{i, \alpha}^{x}\right)^{2}=0 \text {. }
$$

Functions of the $\psi$ 's are determined by their Taylor series. The Berezin integral is defined by

$$
\int \prod_{i} d \psi_{i, u} d \psi_{i, u}^{+} d \psi_{i, d} d \psi_{i, d}^{+} \prod_{i} \psi_{i, u} \psi_{i, u}^{+} \psi_{i, d} \psi_{i, d}^{+}=1
$$

The integrals which involve the same variables in a different order equal \pm 1 , where the sign is calculated through (6.1). Any other integral vanishes.

The measure of integration (6.3) will be noted $\int d \psi d \psi^{+}$. Notice that the sign convention (6.3) is invariant under permutation of the site labels as well as exchange of $u$ and $d$ labels.

We now want to consider the state model expansion $Z_{\text {loop }}$ (Sect. 3) as the graphical expansion of the integral

$$
\int d \psi d \psi^{+} e^{\mathscr{A}}
$$

where $\mathscr{A}$ is some action to be determined. Each state in (3.3) should then correspond to a term in the Taylor expansion of $e^{\mathscr{A}}$ with non-vanishing Berezin integral calculated through the above rules. For this purpose each fermionic edge state of (3.3) (represented by a heavy bond) is interpreted as arising from a "propagator" term. If following the link orientation the edge starts at vertex $i$ and ends at vertex $j$, the propagator term will be $\psi_{i, \alpha}^{+} \psi_{j, \beta}$, where labels $\alpha, \beta=u$ or $d$ depending on the particular configuration
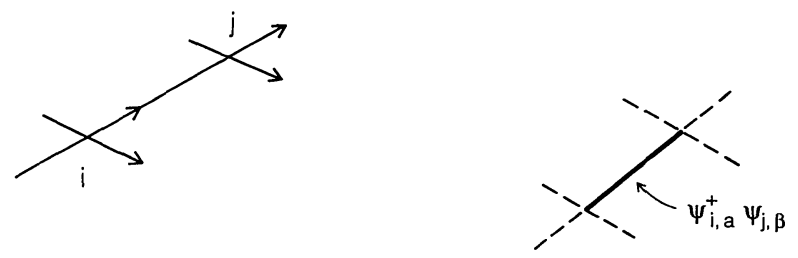

$\mathscr{A}$ thus has a first part

$$
\mathscr{A}_{\text {propagator }} \equiv \mathscr{A}_{p}=\sum_{\substack{\text { oriented edges } \\\langle i, j\rangle}} \psi_{i, \alpha}^{+} \psi_{j, \beta}
$$

Example. Trefoil
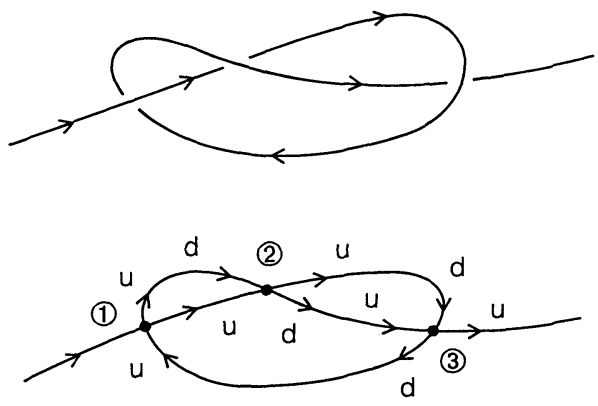

$$
\mathscr{A}_{p}=\psi_{1, u}^{+} \psi_{2, d}+\psi_{1, d}^{+} \psi_{2, u}+\psi_{2, u}^{+} \psi_{3, d}+\psi_{2, d}^{+} \psi_{3, u}+\psi_{3, d}^{+} \psi_{1, u}
$$


Unknot
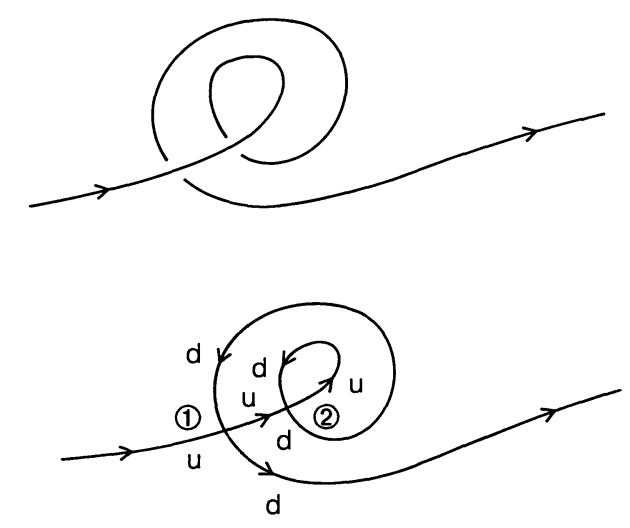

$$
\mathscr{A}_{p}=\psi_{1, u}^{+} \psi_{2, u}+\psi_{2, u}^{+} \psi_{2, d}+\psi_{2, d}^{+} \psi_{1, d}
$$

We have

$$
e^{\mathscr{A}_{p}}=\prod_{\langle i, j\rangle}\left(1+\psi_{i, \alpha}^{+} \psi_{j, \beta}\right)
$$

where the order does not matter since products of an even number of fermions commute. Each term in expansions of (6.6) thus gives rise to a graph with some dashed and some full bonds.

We now come to interactions. First we factor and $q^{\text {\# negative-\# positive crossings }}$ $\left(\equiv q^{-e}\right)$ to get the vertex weights

$\aleph$\begin{tabular}{|c|c|c|c|c|c|}
\hline$\because$ & $\ddots$ & $\searrow$ & $\%$ & $\searrow$ & $X$ \\
\hline$q^{2}$ & $q^{2}-1$ & $q$ & $a$ & 0 & -1 \\
$q^{-2}$ & 0 & $q^{-1}$ & $q^{-1}$ & $q^{-2}-1$ & -1 \\
$w_{1}$ & $w_{5}$ & $w_{4}$ & $w_{3}$ & $w_{6}$ & $w_{2}$ \\
\hline
\end{tabular}

A vertex like
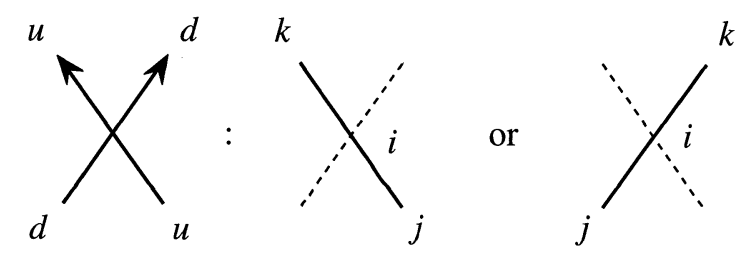

is associated in the propagator expansion to

$$
\psi_{j, \alpha}^{+} \psi_{i, u} \psi_{i, u}^{+} \psi_{k, \beta} \quad \text { or } \psi_{j, \alpha}^{+} \psi_{i, d} \psi_{i, d}^{+} \psi_{k, \beta} \text {. }
$$

These terms contain only two of the four Grassmann variables associated to the vertex $i$. To get a non-zero result after Berezin integration we must then supplement $\mathscr{A}$ by an interaction part that contains $\psi_{i, d} \psi_{i, d}^{+}$(respectively $\psi_{i, u} \psi_{i, u}^{+}$). These terms come naturally with weight $w_{4}$ (respectively $w_{3}$ ). 
In a similar way the vertices
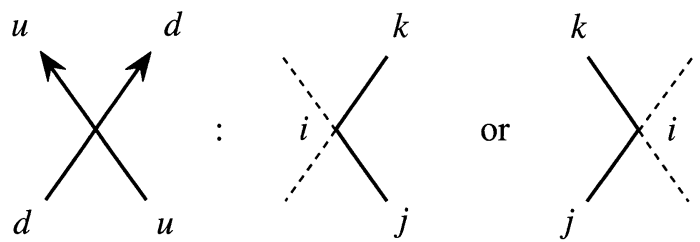

are associated in the propagator expansion to

$$
\psi_{j, \alpha}^{+} \psi_{i, u} \psi_{i, d}^{+} \psi_{k, \beta} \quad \text { or } \quad \psi_{j, \alpha}^{+} \psi_{i, d} \psi_{i, u}^{+} \psi_{k, \beta},
$$

again containing two of the four $i$ fermions only. Hence we must add to $\mathscr{A}$ another interaction part with $\psi_{i, u}^{+} \psi_{i, d}$ (respectively $\psi_{i, d}^{+} \psi_{i, u}$ ). These terms come with weights $w_{5}$ (respectively $w_{6}$ ). So far we have collected

$$
\mathscr{A}_{\text {interaction }} \equiv \mathscr{A}_{I}=\sum_{i} w_{3} \psi_{i, u} \psi_{i, u}^{+}+w_{4} \psi_{i, d} \psi_{i, d}^{+}+w_{5} \psi_{i, u}^{+} \psi_{i, d}+w_{6} \psi_{i, d}^{+} \psi_{i, u}
$$

For a positive crossing

$$
\mathscr{A}_{I}=q\left(\psi_{u} \psi_{u}^{+}+\psi_{d} \psi_{d}^{+}\right)+\left(q^{2}-1\right) \psi_{u}^{+} \psi_{d},
$$

and for a negative crossing

$$
\mathscr{A}_{I}=q^{-1}\left(\psi_{u} \psi_{u}^{+}+\psi_{d} \psi_{d}^{+}\right)+\left(q^{-2}-1\right) \psi_{d}^{+} \psi_{u} .
$$

Now look at the purely bosonic vertex

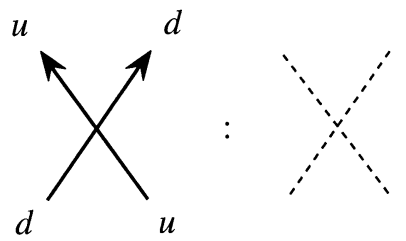

which arises from terms in $\mathscr{A}_{I}$ only. There are two ways to get the 4 Grassmann variables at site $i$, either by combining the two first or two last terms of (6.8),

$$
\begin{gathered}
w_{3} w_{4} \psi_{i, u} \psi_{i, u}^{+} \psi_{i, d} \psi_{i, d}^{+}+w_{5} w_{6} \psi_{i, u}^{+} \psi_{i, d} \psi_{i, d}^{+} \psi_{i, u} \\
=\left(w_{3} w_{4}-w_{5} w_{6}\right) \psi_{i, u} \psi_{i, u}^{+} \psi_{i, d} \psi_{i, d}^{+}
\end{gathered}
$$

due to (5.3) the prefactor is correctly $w_{1}$.

Let us forget for a while the purely fermionic vertices. Then collecting (6.6) and (6.8) the terms with non-zero Berezin integrals are in one-to-one correspondence with the states of the state model such that no loop intersection occurs. The right weight at each crossing is reproduced by the prefactors in $\mathscr{A}_{I}$. The only thing we have to worry about are global signs. The fully empty state comes with

$$
q^{-e} \prod_{i} q^{ \pm 2} \psi_{i, u} \psi_{i u}^{+} \psi_{i, d} \psi_{i, d}^{+}
$$

hence contributes $q^{e}$ to $Z_{\text {loop }}$ as it should. Now suppose we have one loop. For each vertex that does not touch the loop we have $q^{ \pm 2} \psi_{i, u} \psi_{i, u}^{+} \psi_{i, d} \psi_{i, d}^{+}$. For a vertex like
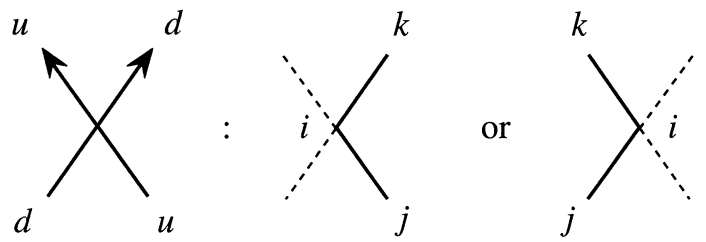
we get

$$
\psi_{j, \alpha}^{+} \psi_{i, u} \psi_{i, d}^{+} \psi_{k, \beta} \psi_{i, u}^{+} \psi_{i, d}=\psi_{i, u} \psi_{i, u}^{+} \psi_{i, d} \psi_{i, d}^{+} \psi_{j, \alpha}^{+} \psi_{k, \beta}
$$

or

$$
\psi_{j, \alpha}^{+} \psi_{i, d} \psi_{i, u}^{+} \psi_{k, \beta} \psi_{i, d}^{+} \psi_{i, u}=\psi_{i, u} \psi_{i, u}^{+} \psi_{i, d} \psi_{i, d}^{+} \psi_{j, \alpha}^{+} \psi_{k, \beta}
$$

For a vertex like

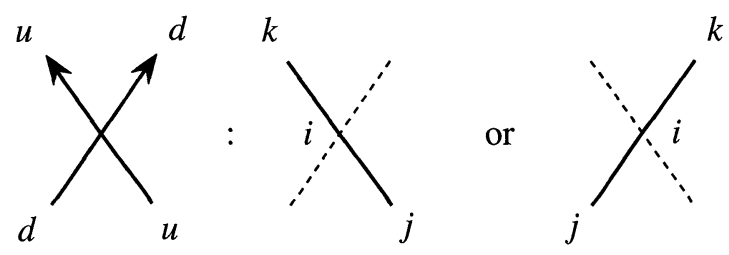

we get

$$
\psi_{j, \alpha}^{+} \psi_{i, u} \psi_{i, u}^{+} \psi_{k, \beta} \psi_{i, d} \psi_{i, d}^{+}=\psi_{i, u} \psi_{i, u}^{+} \psi_{i, d} \psi_{i, d}^{+} \psi_{j, \alpha}^{+} \psi_{k, \beta}
$$

or

$$
\psi_{j, \alpha}^{+} \psi_{i, d} \psi_{i, d}^{+} \psi_{k, \beta} \psi_{i, u} \psi_{i, u}^{+}=\psi_{i, u} \psi_{i, u}^{+} \psi_{i, d} \psi_{i, d}^{+} \psi_{j, \alpha}^{+} \psi_{k, \beta}
$$

Going along the loop we thus factorize out $\psi_{i, u} \psi_{i, u}^{+} \psi_{i, d} \psi_{i, d}^{+}$with the right Boltzmann weight. If we started the loop analysis at some vertex $i$ we get in the end
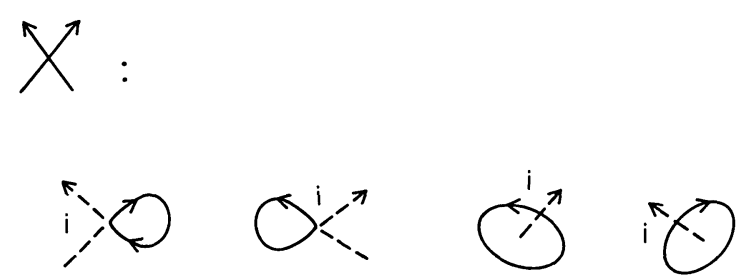

$\psi_{i, d}^{+} \psi_{i, u} \psi_{i, u}^{+} \psi_{i, d} \quad \psi_{i, u}^{+} \psi_{i, d} \psi_{i, d}^{+} \psi_{i, u} \quad \psi_{i, u}^{+} \psi_{i, u} \psi_{i, d} \psi_{i, d}^{+} \quad \psi_{i, d}^{+} \psi_{i, d} \psi_{i, u} \psi_{i, u}^{+}$

equal to $-\psi_{i, u} \psi_{i, u}^{+} \psi_{i, d} \psi_{i, d}^{+}$. Hence the one-loop diagram comes with

$$
-\prod_{i} \psi_{i, u} \psi_{i, u}^{+} \psi_{i, d} \psi_{i, d}^{+} \prod \text { Boltzmann weights, }
$$

reproducing the right sign of the state model. The argument generalizes immediately to a diagram with several non-intersecting fermionic loops each carrying a factor $(-1)$.

Let us finally come to the case where there are fermionic crossings. The vertex
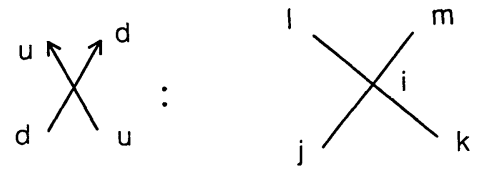
arises from $\mathscr{A}_{p}$ only

$$
\begin{aligned}
\psi_{j, \alpha}^{+} \psi_{i, d} \psi_{i, d}^{+} \psi_{m, \beta} \psi_{k, \gamma}^{+} \psi_{i, u} \psi_{i, u}^{+} \psi_{l, \delta} & =\psi_{i, u} \psi_{i, u}^{+} \psi_{i, d} \psi_{i, d}^{+} \psi_{j, \alpha}^{+} \psi_{m, \beta} \psi_{k, \gamma}^{+} \psi_{l, \delta} \\
& =-\psi_{i, u} \psi_{i, u}^{+} \psi_{i, d} \psi_{i, d}^{+} \psi_{j, \alpha}^{+} \psi_{l, \delta} \psi_{k, \gamma}^{+} \psi_{m, \beta}
\end{aligned}
$$

with respect to the term that would arise from two independent loops (6.11) we get an extra minus sign. This nicely provides the desired value of $w_{2}=-1$. Thus we have

$$
\begin{aligned}
Z_{\text {loop }}= & q^{-e} \int d \psi d \psi^{+} \exp \left\{\sum_{\substack{\text { oriented edges } \\
\langle i, j\rangle}}^{\prime} \psi_{i, \alpha}^{+} \psi_{j, \beta}\right. \\
& +\sum_{\substack{\text { positive } \\
\text { crossings }}} q\left(\psi_{i, u} \psi_{i, u}^{+}+\psi_{i, d} \psi_{i, d}^{+}\right)+\left(q^{2}-1\right) \psi_{i, u}^{+} \psi_{i, d} \\
& +\sum_{\substack{\text { negative } \\
\text { ones }}} q^{-1}\left(\psi_{i, u} \psi_{i, u}^{+}+\psi_{i, d} \psi_{i, d}^{+}\right)+\left(q^{-2}-1\right) \psi_{i, d}^{+} \psi_{i, u}
\end{aligned}
$$

$\sum^{\prime}$ sums over all edges except the one opened to get a tangle. The opened edge carries a boson (0). Writing the action

$$
\mathscr{A}=\sum_{\substack{i, j \\ \alpha, \beta=u, d}} \psi_{i, \alpha i, \alpha} A_{j, \beta} \psi_{j, \beta}^{+}
$$

where $A$ is $(2 \times \# \text { crossings })^{\otimes 2}$ we get

$$
Z_{\text {loop }}=q^{-e} \operatorname{det} A
$$

and

$$
\nabla_{L}=q^{-\operatorname{rot}(L)-e(L)} \operatorname{det} A .
$$

Example. For the trefoil we have

$$
\mathscr{A}_{I}=q \sum_{i=1}^{3}\left(\psi_{i, u} \psi_{i, u}^{+}+\psi_{i, d} \psi_{i, d}^{+}\right)+\left(q^{2}-1\right) \sum_{i=1}^{3} \psi_{i, u}^{+} \psi_{i, d}
$$

Hence

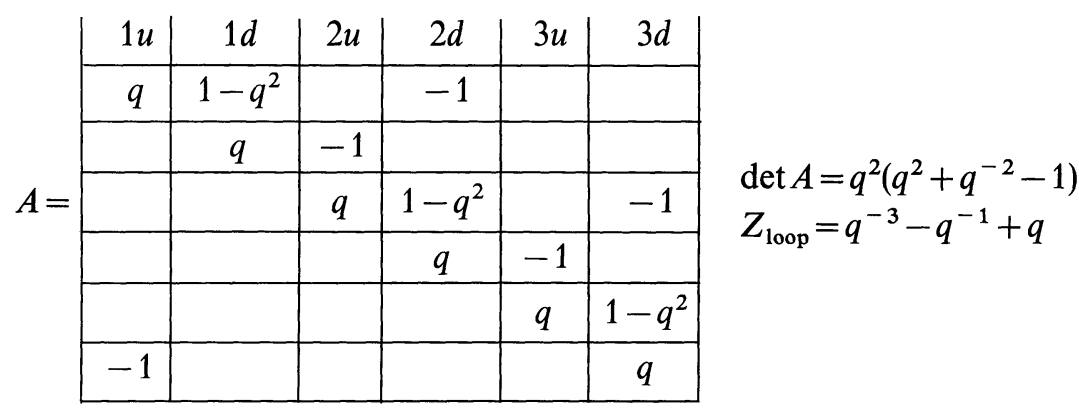

$$
\begin{aligned}
& \operatorname{rot}(L)=-1 \Rightarrow \nabla_{T}=q^{2}+q^{-2}-1=1+z^{2} \text {. }
\end{aligned}
$$


Another way of looking at the trefoil is
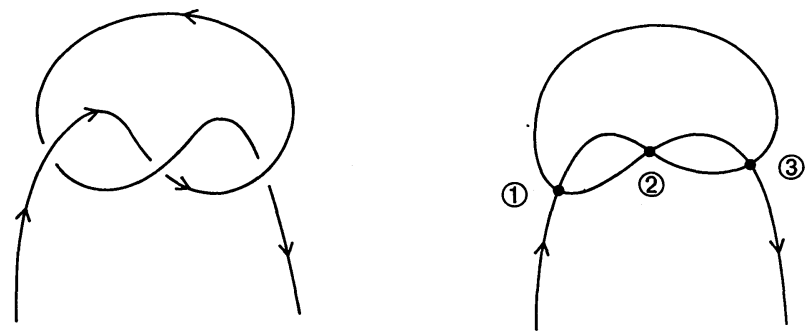

$$
\mathscr{A}_{I}=\psi_{1, u}^{+} \psi_{2, d}+\psi_{1, d}^{+} \psi_{2, u}+\psi_{2, u}^{+} \psi_{3, d}+\psi_{2, d}^{+} \psi_{3, u}+\psi_{3, u}^{+} \psi_{1, d}
$$

$A=$\begin{tabular}{|c|c|c|c|c|c|}
\hline$q$ & $1-q^{2}$ & & -1 & & \\
\hline & $q$ & -1 & & & \\
\hline & & $q$ & $1-q^{2}$ & & -1 \\
\hline & & & $q$ & -1 & \\
\hline & -1 & & & $q$ & $1-q^{2}$ \\
\hline & & & & & $q$ \\
\hline
\end{tabular}

$\operatorname{det} A=q^{4}\left(q^{2}+q^{-2}-1\right)$

$Z_{\text {loop }}=q^{3}-q+q^{-1}$

$$
\operatorname{rot}(L)=1 \Rightarrow \nabla_{T}=q^{2}+q^{-2}-1=1+z^{2} \text {. }
$$

Unknot

$$
\begin{aligned}
& \mathscr{A}_{I}=q^{-1} \sum_{i=1}^{2} \psi_{i, u} \psi_{i, u}^{+}+\psi_{i, d} \psi_{i, d}^{+}+\left(q^{-2}-1\right) \sum_{i=1}^{2} \psi_{i, d}^{+} \psi_{i, u}
\end{aligned}
$$

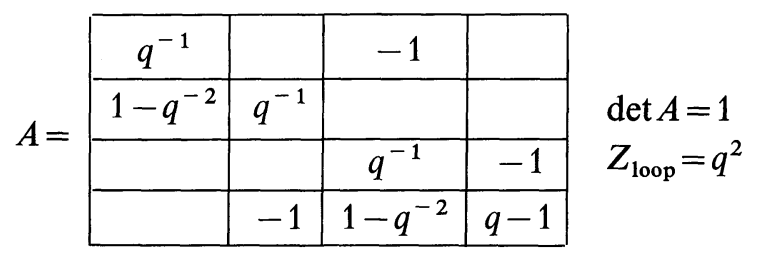

$$
\begin{aligned}
& \operatorname{rot}(L)=2 \Rightarrow \nabla_{L}=1 \text {. }
\end{aligned}
$$

\section{Conclusion}

It has been the purpose of this paper to place the Alexander-Conway polynomial in the context of the $s l(n / n)$ quantum groups and the free-fermion model. This has led to a new description of this link invariant as a determinant for free fermions. Many questions remain for the relationship of these concerns both with the theory of knots and links, and with mathematical physics. A significant hint of deeper relationships is the fact that the Alexander polynomial is a form of Reidemeister torsion for the link complement [M62], and that Witten's theory [W89] includes Reidemeister torsion of the associated three-manifold in the large $k$ limit. This is not a direct connection, but rather an indication that the key question here is the relationship of Reidemeister torsion and mathematical physics. 


\section{Appendix}

Determinant and Trace. It is the purpose of this Appendix to prove the formula

$$
\operatorname{det}(A+s I)=\operatorname{tr}(\mathscr{S} \hat{A}),
$$

where $\hat{A}$ denotes the extension of $A: V \rightarrow V$ to the exterior algebra $\wedge *(V)$ :

$$
\hat{A}: \wedge^{*}(V) \rightarrow \wedge^{*}(V) \text {. }
$$

To this end, let $\left\{v_{1}, v_{2}, \ldots, v_{n}\right\}$ be a basis for $V$ and define $\mathscr{S}: \wedge^{*}(V) \rightarrow \wedge^{*}(V)$ by $\mathscr{S}\left(v_{i 1} \wedge v_{i 2} \wedge \ldots \wedge v_{i k}\right)=s^{k} v_{i 1} \wedge \ldots \wedge v_{i k}$. We are given that

$$
A v_{i}=\sum_{k} A_{k i} v_{k} \text {. }
$$

Now $\wedge *(V)$ has for a basis the elements $v_{i 1} \wedge v_{i 2} \wedge \ldots \wedge v_{i k}$ with $i_{1}<i_{2}<\ldots<i_{k}$ (including 1 for the case $k=0$ ).

Let $\vec{l}=\left(l_{1}, l_{2}, \ldots, l_{k}\right)$ for $l_{1}<l_{2}<\ldots<l_{k}$ and let $\pi(\vec{l})$ denote the set of permutations of the set $\left\{l_{1}, l_{2}, \ldots, l_{k}\right\}$. Given $\pi \in \pi(\bar{l})$, let $\varepsilon(\pi)$ denote the sign of this permutation relative to the convention that $\varepsilon\left(l_{1}, l_{2}, \ldots, l_{k}\right)=1$ when $l_{1}<l_{2}<\ldots<l_{k}$.

Recall that $\hat{A}: \wedge^{*}(V) \rightarrow \wedge^{*}(V)$ is defined via the equation

$$
\hat{A}\left(v_{i 1} \wedge \ldots \wedge v_{i k}\right)=\left(A v_{i 1}\right) \wedge \ldots \wedge\left(A v_{i k}\right) \text {. }
$$

\section{Lemma 1}

$$
\widehat{A} v_{\vec{i}}=\sum_{\hat{l}} \hat{A}_{\vec{l} i} e_{\vec{l}}
$$

where $\vec{i}$ and $\vec{l}$ are multi-indices as described above, and

$$
\hat{A}_{\vec{l} \vec{i}}=\sum_{\pi \in \pi(\vec{l})} \varepsilon(\pi) A_{\pi_{1} i_{1}} \ldots A_{\pi_{k} i_{k}} .
$$

Proof.

$$
\begin{aligned}
\hat{A}\left(v_{i 1} \wedge \ldots \wedge v_{i k}\right) & =\left(A v_{i 1}\right) \wedge\left(A v_{i 2}\right) \wedge \ldots \wedge\left(A v_{i k}\right) \\
& =\left(\sum_{l_{1}} A_{l_{1} i_{1}} v_{l_{1}}\right) \wedge \ldots \wedge\left(\sum_{l_{k}} A_{l_{k} i_{k}} v_{l_{k}}\right) \\
& =\sum_{l_{1}, l_{2}, \ldots, l_{k}} A_{l_{1} i_{1}} \ldots A_{l_{k} i_{k} i_{l_{1}}} v_{l_{1}} \wedge \wedge v_{l_{k}} \\
& =\sum_{l_{1}<\ldots<l_{k}}\left[\sum_{\pi \in \pi(\vec{l})} \varepsilon(\pi) A_{\pi_{1} i_{1}} \ldots A_{\pi_{k} i_{k}}\right] v_{l_{1}} \wedge \ldots \wedge v_{l_{k}}
\end{aligned}
$$

This completes the proof.

Thus we see that the diagonal elements of the matrix $\hat{A}$ take the form

$$
\hat{A}_{\vec{l} \vec{l}}=\sum_{\pi \in \pi(\vec{l})} \varepsilon(\pi) A_{\pi_{1} l_{1}} \ldots A_{\pi_{k} l_{k}} .
$$

In other words $\hat{A}_{\vec{l} \vec{l}}$ is the determinant of the $k \times k$ block in $A$ that is obtained by dropping out the complement of the set of indices $\left\{l_{1}, l_{2}, \ldots, l_{k}\right\}$.

Now compare this observation with the form of the determinant for $A+s I$ :

$$
\operatorname{det}(A+s I)=\sum_{\pi} \varepsilon(\pi) \prod_{k=1}^{n}\left(A_{k \pi_{k}}+s \delta_{k \pi_{k}}\right) \text {. }
$$


It is easy to see, by directly expanding the products in this formula, that

$$
\operatorname{det}(A+s I)=\sum_{\vec{i}} s^{|\vec{l}|} \hat{A}_{\vec{l} \vec{\imath}},
$$

where $|\vec{l}|=k$ when $\vec{l}=\left(l_{1}, l_{2}, \ldots, l_{k}\right)$ with $l_{1}<l_{2}<\ldots<l_{k}$. This is equivalent to the statement that

$$
\operatorname{det}(A+s I)=\operatorname{tr}(\mathscr{S} \hat{A}) .
$$

Hence we are done.

Acknowledgements. It gives us great pleasure to acknowledge useful conversations with Louis Crane, Peter Freund, Bernard Grossman, Francois Jaeger, Vaughan Jones, Frank Nijhoff, and Prasanta Panigrahi. H.S. thanks the Enrico Fermi Institute for its generous hospitality.

\section{References}

[A23] Alexander, J.W.: Trans. Am. Math. Soc. 30, 275-306 (1923)

[AW87] Akutsu, Y., Wadati, W.: J. Phys. Soc. Jpn. 56, 839-842 (1987); J. Phys. Soc. Jpn. 56, 3039-3051 (1987)

[BB80] Balantehin, B., Bars, I.: J. Math. Phys. 22 (1981)

[B74] Birman, J.S.: Braids, links and mapping class groups. Ann. Math. Study 82 (1974), Princeton Univ. Press

[C70] Conway, J.H.: In: Computational problems in abstract algebra. pp. 329-358. New York: Pergamon Press 1970

[CK90] Chaichian, M., Kulish, P.P.: Phys. Lett. B 234, 72 (1990)

[CO89] Corrigan, E., Fairlie, D.B., Fletcher, P., Sasaki, R.: J. Math. Phys.

[D89] Deguchi, T.: J. Phys. Soc. Jpn. 58, 3441 (1989)

[D86] Drinfeld, V.G.: Quantum groups. Proc. Intl. Congress Math., Berkeley 789 (1986)

[DA90] Deguchi, T., Akutsu, Y.: J. Phys. A 23, 1861 (1990)

[DJ81] Dandi, P.H., Jarvis, P.B.: J. Phys. A 14, 547 (1981)

[F63] Fox, R.H., Crowell, R.H.: Introduction to Knot Theory. Blaisdell 1963

[Ja87] Jaeger, F.: Composition products and models for the homfly polynomial. Preprint 87

[J87] Jimbo, M.: Lett. Math. Phys. 10, 63 (1985)

[J86] Jimbo, M.: Commun. Math. Phys. 102, 537 (1986)

[Jo85] Jones, V.F.R.: Bull. Am. Math. Soc. 12, 102-112 (1985)

[Jo87] Jones, V.F.R.: Ann. Math. 126, 335-388 (1987)

[Jo89] Jones, V.F.R.: Pacific J. Math. 137, 189 311-334 (1989)

[JY82] Joyce, D.: J. Pure Appl. Algebra 23, 37-65 (1982)

[Ka77] Kac, V.G.: Commun. Math. Phys. 53, 31 (1977)

[K83] Kauffman, L.H.: Formal Knot Theory. Princeton University Press Math. Notes 30 (1983)

[K1,87] Kauffman, L.H.: On Knots. Ann. Math. Study 15, Princeton University Press 1987

[K2, 87] Kauffman, L.H.: Topology 26, 395-407 (1987)

[K88] Kauffman, L.H.: Contemp. Math., Vol. 78. Am. Math. Soc. pp. 263-297 (1988), and New problems, methods and techniques in quantum field theory and statistical mechanics, pp. 175-222. Singapore: World Scientific 1990

[K1, 89] Kauffman, L.H.: Contemp. Math. 96. Am. Math. Soc. 221-231 (1989)

$[\mathrm{K} 2,89]$ Kauffman, L.: Knots, abstract tensors, and the Yang Baxter equation. In: Knots topology and quantum field theories, pp. 179-334. Singapore: World Scientific 1989

[K90] Kauffman, L.H.: Trans. Am. Math. Soc. 318 (2), 417-471 (1990)

[K91] Kauffman, L.H.: Knots and Physics. Singapore: World Scientific 1991

[KR89] Kulish, P.P., Reshetikhin, N.Yu.: Lett. Math. Phys. 18, 143 (1989)

[KS82] Kulish, P.P., Sklyanin, E.K.: J. Sov. Math. 19, 1596 (1982)

[KS91] Kauffman, L., Saleur, H.: Fermions and link invariants. Preprint YCTP-P21-91

[LCS88] Lee, H.C., Couture, M., Schmeling, N.C.: Connected link polynomials. Preprint 88 
[LiM87] Lickorish, W.B.R., Millett, K.: Topology 26, 107 (1987)

[LW71] Lieb, E., Wu, F.Y.: In: Phase transitions and critical phenomena, Vol. 1. Domb, C., Green, M.S. (eds.). New York: Academic Press 1971

[M62] Milnor, J.W.: Ann. Math. 76, 137-147 (1962)

[PS90] Pasquier, V., Saleur, H.: Nucl. Phys. B 330, 523 (1990)

[R87] Reshetikhin, N.Y.: Quantized universal enveloping algebras. Preprint, LOMI E-4-87, E-17-87, Steklov Institute, Leningrad

[R89] Reshetikhin, N.Yu.: Unpublished

[S89] Saleur, H.: Symmetries of the XX chain and applications. In: Proceedings of recent developments in conformal field theories. Trieste, Oct. 2-Oct. 4, 1989. Singapore:

[S90] Saleur, H.: Nucl. Phys. B 336, 363 (1990)

[Sa80] Samuel, S.: J. Math. Phys. 21, 2806 (1980)

[Sc79] Scheunert, M.: The theory of Lie superalgebras. Lect. Notes in Mathematics, Vol. 716. Berlin, Heidelberg, New York: Springer 1979

[SC81] Schultz, C.L.: Phys. Rev. Lett. 46, 629 (1981)

[SVZ89] Schmidke, W., Vokos, S.P., Zumino, B.: Preprint UCB-PTH-89/32

[T88] Turaev, V.G.: Invent. Math. 92, 527-553 (1988)

[WDA89] Wadati, M., Deguchi, T., Akutsu, Y.: Phys. Rep. 180, 247 (1989)

[W89] Witten, E.: Commun. Math. Phys. 121, 351-399 (1989)

Communicated by K. Gawedzki 
